\title{
Voltage-gated potassium channel EAG2 controls mitotic entry and tumor growth in medulloblastoma via regulating cell volume dynamics
}

\author{
Xi Huang, ${ }^{1,2,3}$ Adrian M. Dubuc, ${ }^{4}$ Rintaro Hashizume, ${ }^{5}$ Jim Berg, ${ }^{1,2,3}$ Ye He $^{1,2,3}$ Ji Wang, ${ }^{6}$ \\ Chin Chiang, ${ }^{7}$ Michael K. Cooper, ${ }^{8}$ Paul A. Northcott, ${ }^{4}$ Michael D. Taylor, ${ }^{4}$ Michael J. Barnes, ${ }^{9}$ \\ Tarik Tihan, ${ }^{9}$ Justin Chen, ${ }^{5}$ Christopher S. Hackett, ${ }^{5}$ William A. Weiss, ${ }^{5}$ C. David James, ${ }^{5}$ \\ David H. Rowitch, ${ }^{10,11}$ Marc A. Shuman, ${ }^{12}$ Yuh Nung Jan, ${ }^{1,2,3}$ and Lily Yeh Jan ${ }^{1,2,3,13}$ \\ ${ }^{1}$ Howard Hughes Medical Institute, ${ }^{2}$ Department of Physiology, ${ }^{3}$ Department of Biophysics and Biochemistry, University of \\ California at San Francisco, San Francisco, California 94158, USA; ${ }^{4}$ Division of Neurosurgery, Arthur and Sonia Labatt Brain \\ Tumor Research Center, Hospital for Sick Children, Toronto, Ontario, M5G 1X8, Canada; ${ }^{5}$ Department of Neurological Surgery, \\ ${ }^{6}$ Department of Urology, UCSF Helen Diller Comprehensive Cancer Center, University of California at San Francisco, San \\ Francisco, California 94158, USA; ${ }^{7}$ Department of Cell and Developmental Biology, ${ }^{8}$ Department of Neurology, Vanderbilt \\ University Medical Center, Nashville, Tennessee 37232, USA; ${ }^{9}$ Department of Pathology, UCSF Helen Diller Comprehensive \\ Cancer Center, University of California at San Francisco, San Francisco, California 94158, USA; ${ }^{10}$ Howard Hughes Medical \\ Institute, ${ }^{11}$ Department of Neurological Surgery, University of California at San Francisco, San Francisco, California 94143, \\ USA; ${ }^{12}$ Department of Medicine, UCSF Helen Diller Comprehensive Cancer Center, University of California at San Francisco, \\ San Francisco, California 94158, USA
}

Medulloblastoma (MB) is the most common pediatric CNS malignancy. We identify EAG2 as an overexpressed potassium channel in MBs across different molecular and histological subgroups. EAG2 knockdown not only impairs MB cell growth in vitro, but also reduces tumor burden in vivo and enhances survival in xenograft studies. Mechanistically, we demonstrate that EAG2 protein is confined intracellularly during interphase but is enriched in the plasma membrane during late G2 phase and mitosis. Disruption of EAG2 expression results in G2 arrest and mitotic catastrophe associated with failure of premitotic cytoplasmic condensation. While the tumor suppression function of EAG2 knockdown is independent of p53 activation, DNA damage checkpoint activation, or changes in the AKT pathway, this defective cell volume control is specifically associated with hyperactivation of the p38 MAPK pathway. Inhibition of the p38 pathway significantly rescues the growth defect and G2 arrest. Strikingly, ectopic membrane expression of EAG2 in cells at interphase results in cell volume reduction and mitotic-like morphology. Our study establishes the functional significance of EAG2 in promoting MB tumor progression via regulating cell volume dynamics, the perturbation of which activates the tumor suppressor p38 MAPK pathway, and provides clinical relevance for targeting this ion channel in human MBs.

[Keywords: EAG2; potassium channel; medulloblastoma; cell cycle; mitosis; cell volume]

Supplemental material is available for this article.

Received April 6, 2012; revised version accepted July 9, 2012.

Medulloblastoma (MB), the most common malignant pediatric CNS tumor, is a neuroepithelial malignancy characterized by rapid progression, aggressive growth, and tendency to spread along the leptomeninges of the brain and spinal cord. Standard-of-care treatment with surgery, radiation, and chemotherapy, although successful in just over half of the patients, typically results in serious

\footnotetext{
${ }^{13}$ Corresponding author E-mail lily.jan@ucsf.edu

Article published online ahead of print. Article and publication date are online at http://www.genesdev.org/cgi/doi/10.1101/gad.193789.112.
}

cognitive and neuroendocrine deficits that substantially impact the quality of life.

Recent integrative genomic approaches have identified four MB molecular subgroups (WNT, SHH, group 3, and group 4) with distinct gene expression profiles and clinical presentations (Thompson et al. 2006; Kool et al. 2008; Cho et al. 2011; Northcott et al. 2011b). Cerebellar granule neuron precursors (CGNPs) have been implicated as the cellular origin of MBs initiated in the cerebellum by Sonic hedgehog (Shh) signaling (Schuller et al. 2008; Yang et al. 2008). Shh stimulates CGNP proliferation during development (Weschler-Reya and Scott 1999) involving Mycn and 
D-type Cyclins (Kenney and Rowitch 2000; Ciemerych et al. 2002; Kenney et al. 2003). Mouse models of Shhinduced MBs have been most extensively studied for gaining molecular insight into the SHH subgroup of human MBs. Mouse mutants heterozygous or with cerebellar tissues homozygously floxed for the null mutation of the Shh pathway suppressor Ptch1 (Goodrich et al. 1997; Yang et al. 2008) or expressing a constitutively active form of the Shh pathway activator Smoothened (SmoA1 or SmoM2) (Hatton et al. 2008; Schuller et al. 2008) develop MBs that closely resemble human SHH-MBs. Several Shh-Smo pathway inhibitors have demonstrated encouraging potencies in mouse models and are being tested as therapeutic agents for treating human MBs with SHH pathway activation (Romer and Curran 2005; Rudin et al. 2009; Yauch et al. 2009).

Hedgehog pathway antagonist resistance with relapse (Rudin et al. 2009; Yauch et al. 2009; Buonamici et al. 2010) and bone growth inhibition in pediatric patients (Kimura et al. 2008) underscore the importance of identifying novel molecular targets. Moreover, $\mathrm{SHH}$-activated MBs represent approximately one-quarter of all MBs. The diverse histological presentations and the absence of CGNP markers (Salsano et al. 2004, 2007) or a SHH pathway signature in the majority of MBs mandate the urgency of studying the cellular origins and molecular profiles of non-SHH MBs. Notably, overactivation of the Wnt pathway in mice leads to MBs that arise from the dorsal brain stem (Gibson et al. 2010), whereas overexpressing MYCN in the hindbrain of transgenic mice or orthotopic allografting Myc-overexpressing mouse cerebellar stem cells with mutant p53 resulted in MYCN- or Myc-driven MBs (Swartling et al. 2010; Kawauchi et al. 2012; Pei et al. 2012). Human non-SHH MBs include WNT-activated tumors $(10 \%-15 \%$ of $\mathrm{MBs})$, group 3 (26\% of MBs), and group $4(34 \%$ of $\mathrm{MBs})$, with elevated expression of photoreceptor and neuronal differentiation genes, respectively. It is critically important to identify novel targets that drive $\mathrm{MB}$ cell growth and tumor progression for the majority of MB subgroups.

The contribution of ion channels toward MB tumorigenesis and progression has not been explored. The voltage-gated potassium channel ether-à-go-go (eag), first identified in Drosophila melanogaster based on the legshaking mutant phenotype (Kaplan and Trout 1969; Warmke et al. 1991), has mammalian homologs that fall into three subfamilies-EAG (EAG1 and EAG2), EAGlike (ELK1,ELK2, and ELK3), and EAG-related (ERG1, $E R G 2$, and $E R G 3$ ) - which are voltage-gated potassium channels with distinct electrophysiological properties (Ganetzky et al. 1999). EAG1 (KCNH1, Kv10.1) has been studied for its potential role in cancer (Pardo et al. 1999, 2005; Weber et al. 2006). While its normal expression is largely confined to the CNS, EAG1 is highly expressed in $>75 \%$ of human non-CNS cancers (Hemmerlein et al. 2006; Mello de Queiroz et al. 2006), although the mechanism by which EAG1 stimulates tumor growth is unknown. It is also unknown whether EAG2 (KCNH5, Kv10.2), which displays much higher expression in the cerebral cortex than the cerebellum (Ludwig et al. 2000), has any involvement in cancer. To investigate the molecular mechanisms that drive $\mathrm{MB}$ development and progression, we conducted a genome-wide microarray analysis of mouse MBs and found that the expression of Eag2 was consistently up-regulated. We further confirmed Eag2/EAG2 overexpression in a significant subset of mouse and human MBs across molecular (WNT, SHH, or group 4) and histological (nodular, classic, desmoplastic, or anaplastic) subgroups. Our results demonstrate the importance of the voltage-gated potassium channel EAG2 for promoting $\mathrm{MB}$ cell growth, provide mechanistic insight into its involvement in $\mathrm{MB}$ cell proliferation via cell volume regulation, and identify EAG2 as a potential druggable target in treating human MBs.

\section{Results}

Eag2 is highly up-regulated in Shh-driven mouse MBs

To explore the contribution of ion channels during $M B$ tumorigenesis, we performed microarray analysis on normal adult cerebellum and tumors derived from two Shh-driven mouse MB models (Math1-Cre; SmoM2 [Schuller et al. 2008] and Ptch ${ }^{\text {LacZ/+ }}$ [Goodrich et al. 1997]). Strikingly, Eag2 mRNA expression was increased by $\sim 7.5$-fold in MBs relative to normal cerebellum, while the expression level of its closest family member, Eag1, was decreased by about ninefold (Fig. 1A). The expression levels of several other potassium channels previously shown to be up-regulated in cancers were either significantly decreased (TASK3 [Mu et al. 2003], BK [Liu et al. 2002; Bloch et al. 2007], GIRK1 [Stringer et al. 2001]) or unchanged (Kv1.3 [Fraser et al. 2003]) (Fig. 1A; Supplemental Fig. 1A). In fact, Eag2 was one of the most upregulated ion channel genes in our entire gene array analyses (Fig. 1B; Supplemental Fig. 1B). We validated our microarray results using conventional and quantitative RT-PCR and found significantly increased Eag2 transcript levels in mouse $\mathrm{MB}$ compared with Eag2 expression in normal cerebellum at different developmental stages (Fig. 1C,D). RNA in situ hybridization analyses further demonstrated tumor-specific robust Eag2 expression as compared with the moderate to low expression in adjacent normal tissue or the control cerebella (Fig. 1E).

Consistent with our expression analyses, we detected a striking increase of Eag2 protein in tumor tissues as compared with normal cerebella (Fig. 1F), with prominent Eag2 protein expression in the Ptch ${ }^{\text {LacZ/+ }}$ mouse MB (Fig. 1G; Supplemental Fig. 1C) next to nontumor cerebellar tissues with moderate Eag2 levels (Fig. 1G). In the Math1Cre; SmoM2 mouse MB model with the constitutively active SmoM2 tagged with YFP to mark tumor cells (Mao et al. 2006), robust Eag2 protein expression was evident in MB cells marked by YFP, which also expressed the neural progenitor marker Nestin or the proliferative cell marker Ki67 (Fig. 1G). Importantly, human MB xenograft tumors (Supplemental Fig. 1C) and the CGNPs in the normal cerebellum of P7 (postnatal day 7) wild-type mice (Supplemental Fig. 1D) displayed similar high expression of EAG2/Eag2, while cells in the internal granule neuron 

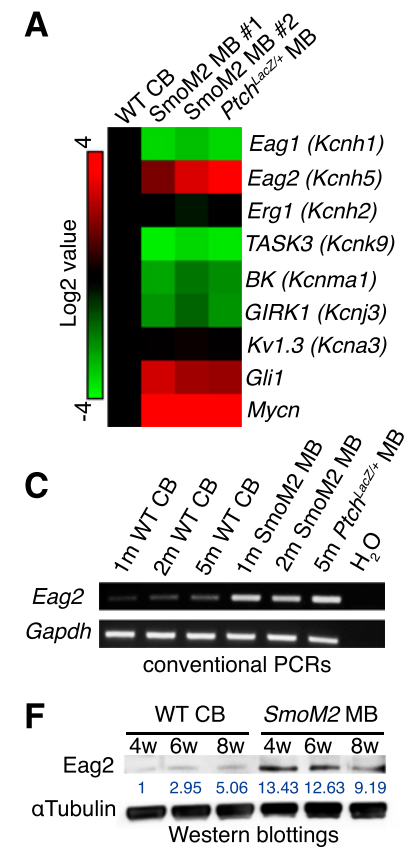

B
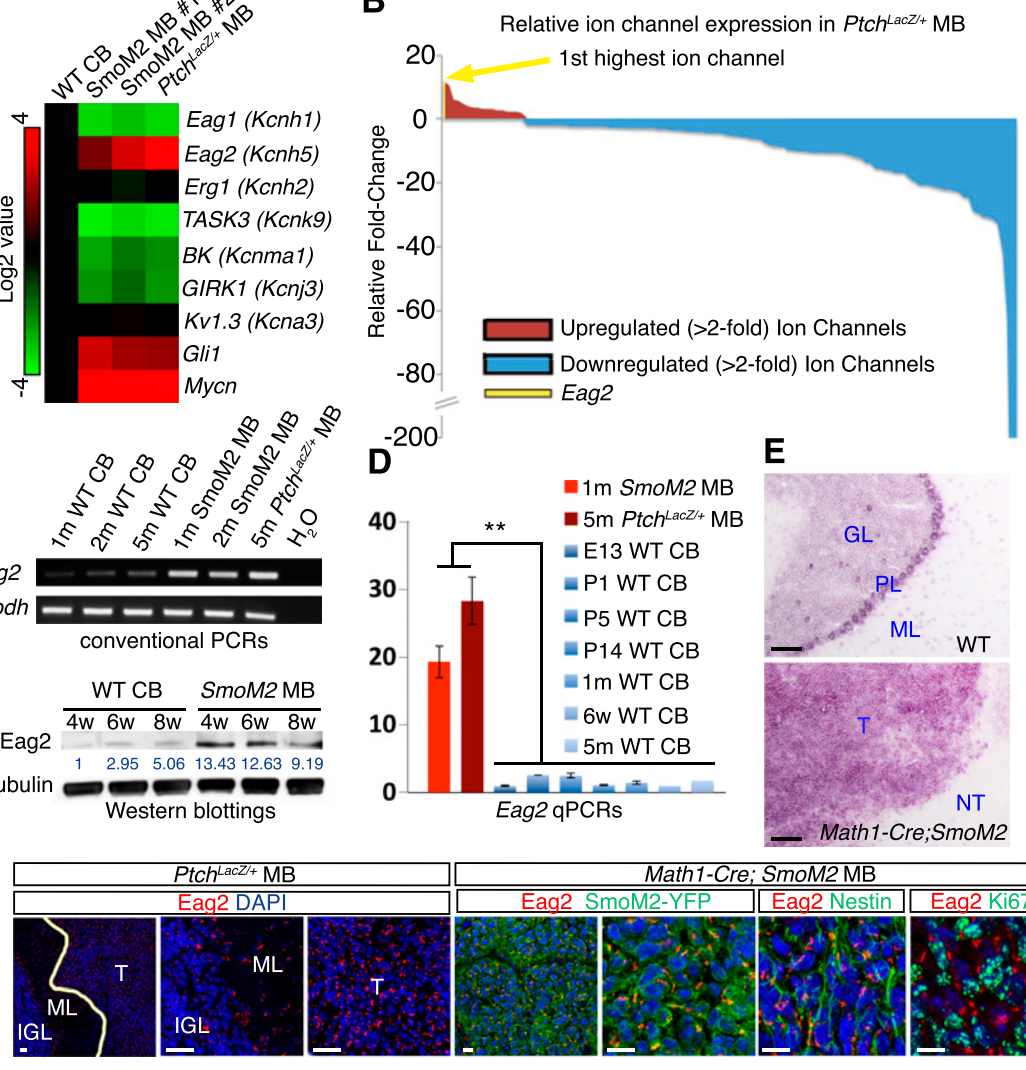

Math1-Cre; SmoM2 MB Math1-Cre

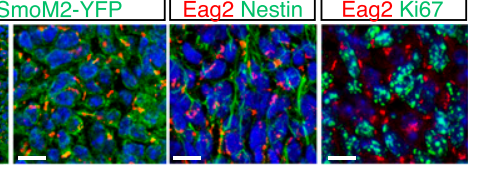

H
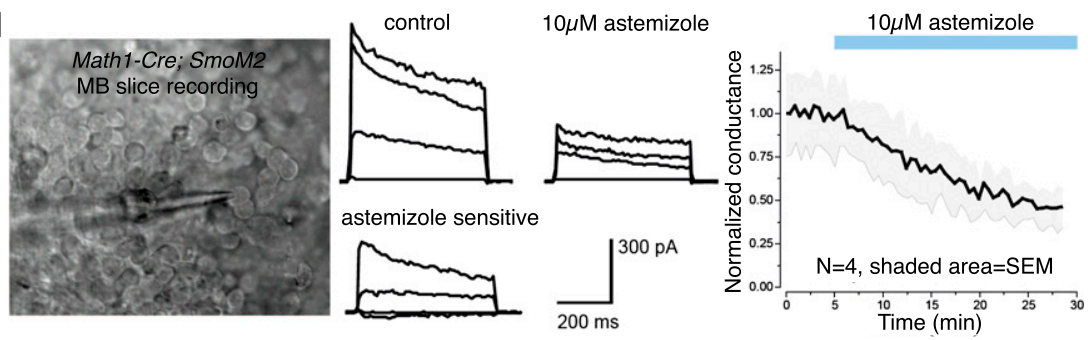

Figure 1. Eag2 is highly overexpressed in Shh-driven mouse MBs. (A) Microarray analyses comparing transcript levels of several potassium channels between MBs and control cerebellum. Shh pathway activation is confirmed by the increased Gli1 and Mycn expression. (B) Relative ion channel gene expression levels in the Ptch $^{\text {LacZ/+ }}$ MB and control cerebellum. $(C)$ Conventional RT-PCRs demonstrate significantly increased Eag2 transcript levels in tumors compared with the normal cerebella of different developmental stages. (D) Quantitative RT-PCRs demonstrate significantly increased Eag2 transcript levels in tumors compared with the normal cerebella of different developmental stages $(N=3$ for each stage). $(E)$ Eag2 RNA in situ hybridization shows moderate expression in the adult cerebellar molecular layer (ML), purkinje layer $(\mathrm{PL})$, and internal granule neuron layer (GL) and dramatic up-regulation in the Math1-Cre; SmoM2 MB tumor (T) tissue but not its adjacent nontumor (NT) tissue. $(F)$ Western blotting shows a marked increase of Eag2 protein in the Math1-Cre; SmoM2 MB tumors $(N=3)$ compared with the age-matched control cerebella $(N=3)$. $(G)$ Eag2 immunostaining shows prominent expression in the MB tumor tissue. $(H)$ Whole-cell voltage clamp recordings of mouse MB cells ex vivo. A representative snapshot of electrode patching a randomly selected MB cell in an acute tumor slice is shown. Whole-cell voltage clamp traces from a MB cell in an acute tumor slice reveal a prominent outward current, a substantial portion of which is sensitive to the EAG2 blocker astemizole. Cells were stepped from a holding potential of $-80 \mathrm{mV}$ to $-40 \mathrm{mV}$ to $+80 \mathrm{mV}$ in $40-\mathrm{mV}$ steps. Application of astemizole effectively reduces the outward conductance in four out of four MB cells tested. $\left(^{\star \star}\right) P<0.001$ by Student's $t$-test. Bars: $E, 250 \mu \mathrm{m} ; G, 25 \mu \mathrm{m}$.

layer displayed low Eag2 expression (Supplemental Fig. 1D).

\section{MB cells display large delayed rectifier voltage-gated potassium channel activity}

To interrogate the functionality of Eag2 channels in $\mathrm{MB}$ cells, we performed whole-cell voltage clamp recordings from randomly selected cells in freshly harvested tissue slices of tumors from Math1-Cre; SmoM2 mice that were older than $1 \mathrm{mo}$ and had highly advanced tumor mass often encompassing most of the cerebellum. At this stage, $\sim 100 \%$ of the tumor cells were marked by SmoM2$\mathrm{YFP}^{+}$, and $\sim 86 \%$ of the cells (1043 of 1210 from three mice) were Ki67 $7^{+}$and proliferating (Fig. 1G). As expected from the abundant Eag2 protein expression in MB cells, pronounced delayed rectifier voltage-gated potassium current was recorded in every tumor cell examined $(N=16)$
(Fig. 1H). The potassium conductance was reduced by $\sim 50 \%$ upon application of the Eag2 channel blocker astemizole $(10 \mu \mathrm{M})$ (Fig. 1H).

\section{EAG2 up-regulation is a hallmark of a subset of human $M B s$}

We further assessed human EAG2 expression in a collection of 12 MBs derived from patients treated in Vanderbilt University Medical Center (Vandy-MB1-12) that encompassed primary and recurrent MBs with extensive nodularity (MBEN) and classic, desmoplastic, and large cell anaplastic (LCA) histologies. (The detailed clinical features are presented in Supplemental Table 1). Compared with pooled RNA from 10 nontumor adult human cerebellar tissues (Clontech) as control, EAG2 expression was markedly elevated in $92 \%$ (11 of 12 ) of MB samples, ranging from an $\sim 6.6$-fold increase in Vandy-MB-2 to 
an 2045 -fold increase in Vandy-MB-12 (Fig. 2A; Supplemental Table 1).

We next turned to a $\mathrm{MB}$ transcriptome data set generated on a large cohort of primary MBs $(n=103)$ and normal human cerebella. While the normal adult cerebella displayed minimal EAG2 expression, we detected up-regulation of EAG2 in 14\% (14 of 103) of MB samples, most prominently in the WNT subgroup (100\%, eight of eight with greater than twofold up-regulation; statistically significant up-regulation in WNT vs. all MBs; $\mathrm{WNT}=7.98 ; \mathrm{MBs}=4.59 ; P<9.13 \mathrm{E}-4$, Mann-Whitney $U$-Test) and also in group $4(11 \%$, four of 31$)$ and $\mathrm{SHH}$ MBs (6\%, two of 33) (Fig. 2B; Supplemental Fig. 2A). In this cohort as well as the Vanderbilt cohort, EAG2 overexpression was notable across multiple molecular subgroups and histological subgroups and over a spectrum of ages (Fig. 2B; Supplemental Table 1). Consistent with the down-regulation of Eag2 during mouse CGNP differentiation (Supplemental Fig. 1D), EAG2 transcript expression in adult human cerebella was significantly reduced compared with that of the fetal cerebella (Fig. 2B). Similar $E A G 2$ expression trends were found in another no-overlapping $\mathrm{MB}$ expression data set $(N=62)$ (Supplemental Fig. 2B; Kool et al. 2008), reconfirming our findings. Interestingly, Eag2 expression was found to be enriched in the Ptch ${ }^{\text {LacZ/+ }}$ MBs but not in the Myc/dominant-negative p53-driven mouse MBs that model the group 3 human MBs with high Npr3 expression (Supplemental Fig. 2C; Pei et al. 2012). These recently published data are in further agreement with our finding that EAG2 transcript upregulation was seen in $\mathrm{WNT}, \mathrm{SHH}$, and group 4 but not group 3 human MBs.

Compared with the EAG2 up-regulation that is prominent in human WNT-MBs and a fraction of the SHH-MBs,

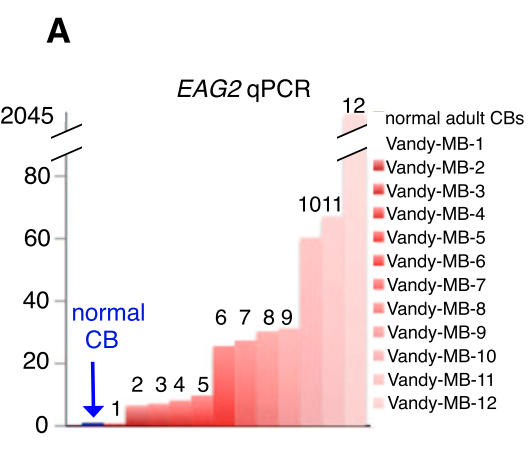

B
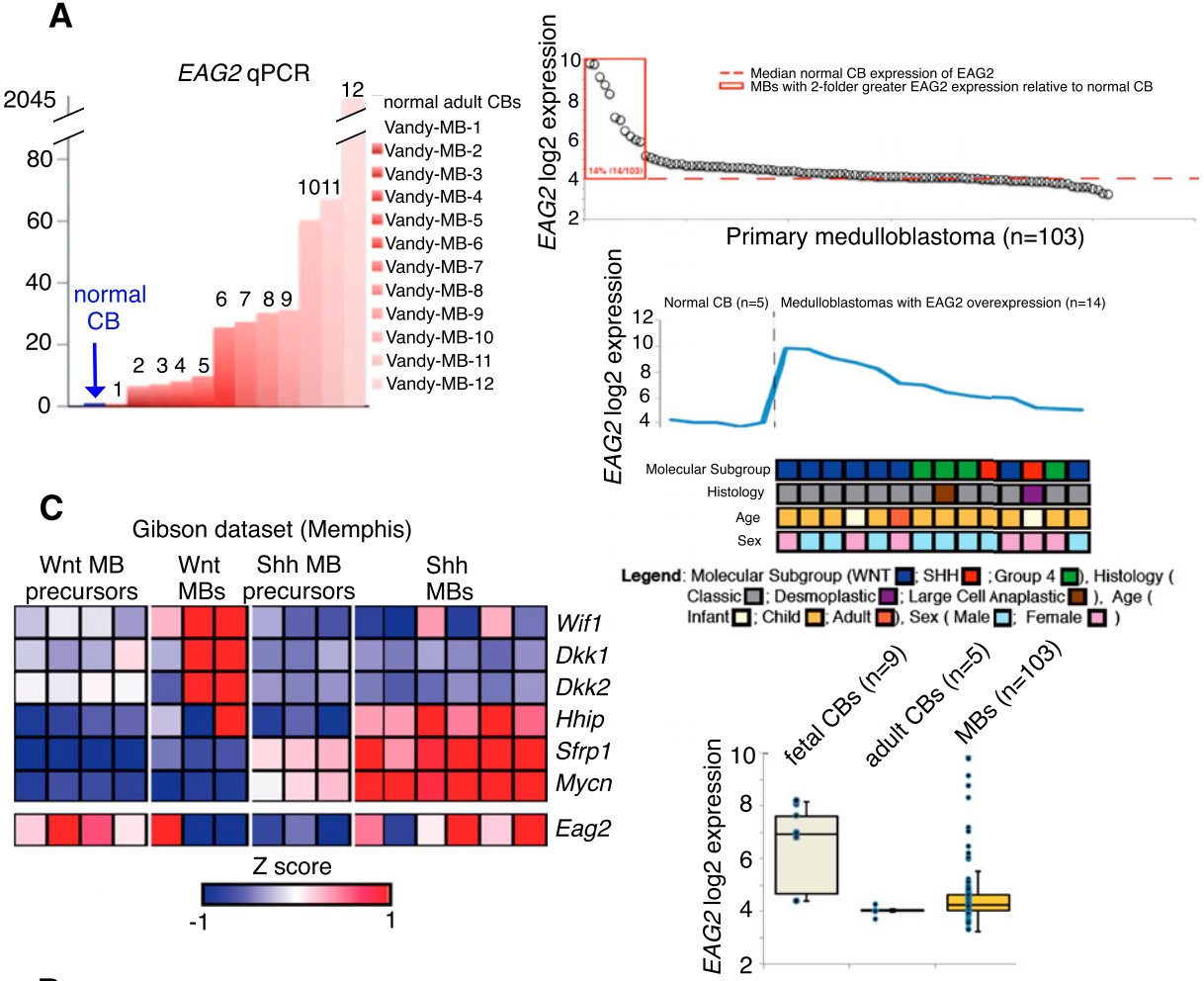

D

human MBs

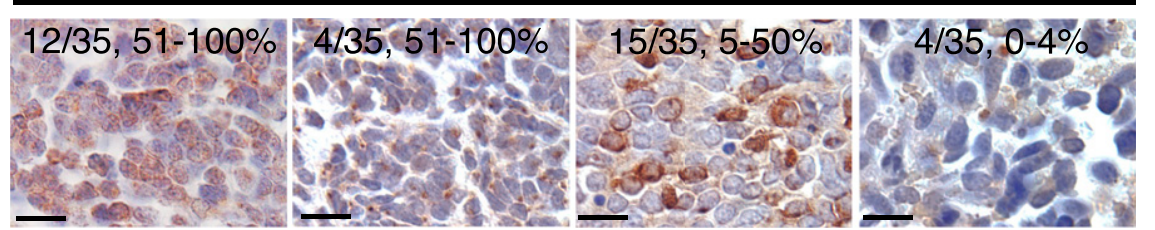

Figure 2. EAG2 up-regulation is a hallmark in subset of human MBs. (A) EAG2 transcript levels are significantly up-regulated in the tumor biopsies in 11 of the $12 \mathrm{MB}$ patients. $(B)$ mRNA microarray analyses in a cohort of $103 \mathrm{MB}$ patient tumor tissues identify significant EAG2 up-regulation in $14 \%$ (14 of 103) of MBs with different molecular and histological subgroups, age, and sex. (C) In previously microarrayed mouse MBs (Gibson et al. 2010), Eag2 increases in a subset of both Shh- and Wnt-driven MBs. (D) Immunohistochemical analyses of EAG2 expression in 35 paraffin-embedded human MB tissues show differential expression patterns. Bar, $D, 20 \mu \mathrm{m}$. 
the robust up-regulation of Eag2 in Shh-driven mouse MBs may reflect species differences. Alternatively, the Shh mouse models may only represent a specific subtype of human SHH-MBs (Northcott et al. 2011a). Consistent with our findings, previously published mouse microarray results showed up-regulation of Eag2 in 50\% (three of six) of Shh-MB mouse models (expressing Shh pathway markers including Hhip, Sfrp1, and Mycn) and only 33\% (one of three) of Wnt-MB mice (expressing Wnt pathway markers including Wif1, Dkk1, and Dkk2) (Fig. 2C; Gibson et al. 2010), although Eag2 was highly expressed in 100\% (four of four) of Wnt precursor cells derived from the lower rhombic lip (Fig. 2C; Gibson et al. 2010). In accordance with these findings, Eag2 protein is abundantly expressed in the lower rhombic lip region of the E16.5 (embryonic day 16.5) wild-type mouse embryo (Supplemental Fig. 1E).

To analyze the subcellular localization of EAG2 protein in human $\mathrm{MB}$ samples, we obtained paraffin sections from 35 additional MB specimens (UCSF-MB1-35, including primary and recurrent tumors with MBEN, classic, desmoplastic, and LCA histologies). (The detailed clinical features and EAG2 expression scores are presented in Supplemental Table 2.) We found robust EAG2 expression, ranging from $51 \%$ to $100 \%$ positivity, in $46 \%$ of the samples ( 16 of 35 ) (Fig. 2D). The remaining $43 \%$ and $11 \%$ of biopsied samples displayed $5 \%-50 \%$ and $0 \%-4 \%$ positivity, respectively (Fig. 2D). While in some cases EAG2 was clearly detected in the MB cell membrane and intracellular compartments, in a subset of MBs, EAG2 was present in distinct punctate structures (Fig. 2D) reminiscent of the pattern in Math1-Cre; SmoM2, Ptch ${ }^{\text {LacZ/+ }}$ mouse MBs and tumor xenografts (Fig. 1G; Supplemental Fig. 1C). Therefore, at the protein level, we found robust EAG2 expression across all of the histological subgroups (Supplemental Table 2), likely representing the majority of molecular subgroups of MB. Taken together, our analyses of EAG2 expression in four independent cohorts of human MBs, totaling 212 tumors, demonstrated various degrees of EAG2 up-regulation in MBs with diverse clinical features and molecular profiles, revealing that EAG2 overexpression is a hallmark in a wide subset of human MBs.

Specific RNAi knockdown of EAG2 impairs MB cell growth in vitro, reduces $M B$ cell tumorigenicity in vivo, and improves the survival of tumor-bearing mice

Although our analyses of EAG2 expression in human MB transcriptome data sets indicate the informative value of determining the functional roles of EAG2 in WNT-MBs, the lack of WNT-MB-derived cell lines (Supplemental Fig. 3) precluded detailed functional studies in this $M B$ subgroup. Thus, to explore the functional roles of EAG2 during MB cell growth in currently available models, we performed both transfection-mediated siRNA and lentivirus-mediated shRNA treatments for knockdown of EAG2 in low-passage primary MB cells (Vandy-MB-11) established from freshly resected patient tumor tissue with very high EAG2 expression (Fig. 2A), Ptch ${ }^{\mathrm{LacZ} /+}$; $p 53^{-1-}$ mouse MB cells, and the DAOY MB cell line. It is of note that the Vandy-MB-11 cells were established from an 11-yr-old male patient who developed MBEN in the cerebellar vermis (Supplemental Table 1) and that the DAOY cells were originally established from a desmoplastic $\mathrm{MB}$, suggesting that these cells likely belonged to the SHH-MB subgroup (McManamy et al. 2007; Ellison et al. 2011). These RNAi treatments selectively reduced the EAG2 expression level but did not affect EAG1 expression (Fig. 3A; Supplemental Figs. 4, 5). Importantly, EAG2 knockdown potently inhibited the growth of not only DAOY cells but also low-passage primary human $\mathrm{MB}$ and $\mathrm{Ptch}^{\mathrm{LacZ/+}} ; \mathrm{p} 53^{-/-}$mouse MB cells by inhibiting proliferation and inducing apoptotic cell death; the same phenotypes were induced using multiple RNAi sequences targeting different regions of the Eag2 transcript (Fig. 3A; Supplemental Fig. 5). Interestingly, we found that the MYCN-driven MBs developed in the GTML mice (Swartling et al. 2010, 2012) displayed a level of Eag2 mRNA expression comparable with that of the normal cerebella at P7 (Supplemental Fig. 6A). We found that the GTML2 cell growth was insensitive to further reduction of the Eag2 expression (Supplemental Fig. 6B). Notably, GTML2 cells were also insensitive to the Smoothened antagonist cycloplamine (Swartling et al. 2012), indicating that they were derived from a non-Shh-MB. Taken together, these findings demonstrate that EAG2 and its mouse homolog confer a growth advantage to those MBs with elevated expression of this potassium channel by promoting proliferation and survival.

To test whether EAG2 knockdown suppresses the oncogenic potential of $\mathrm{MB}$ cells in vivo, we performed supratentorial injections of 150,000 firefly luciferaseexpressing Vandy-MB-11 cells (treated with either scrambled or EAG2 shRNA by lentiviral infection) into the brains of $\mathrm{nu} / \mathrm{nu} B A L B / C$ mice and monitored tumor growth over time by noninvasive bioluminescence imaging (BLI). While tumors from each treatment group displayed similar BLI readings $6 \mathrm{~d}$ after injection (Fig. 3C), EAG2 knockdown profoundly slowed the tumor growth rate (Fig. 3C). Correspondingly, mice bearing tumors with EAG2 knockdown survived twice as long as the control mice $(N=9$, median survival time $104 \mathrm{~d}$ for EAG2 knockdown as compared with $43 \mathrm{~d}$ for control, $P<0.0001$ ) (Fig. 3D). Strikingly, six of the nine $(66 \%)$ control mice developed metastases to the spinal cord, a cardinal feature of malignant $\mathrm{MB}$ growth, whereas none of the tumors with EAG2 knockdown could be detected outside the brain (Fig. 3B). We further performed histological analyses on the brains dissected from the control and EAG2 knockdown tumors. Consistent with the BLI results, we found massive growth of control tumors encompassing the majority of the brain hemisphere at the injection site (Fig. 3B), while the tumors with EAG2 knockdown were considerably smaller, although with similar histopathology. In agreement with the xenograft studies using primary MB cells, our study of DAOY cell xenografts also revealed significant inhibition of tumor growth in vivo by EAG2 knockdown (Fig. 3E,F). The tumors with EAG2 knockdown displayed significantly reduced in vivo growth; nevertheless, all of the mice eventually succumbed to disease, indicating tumor engraftment 

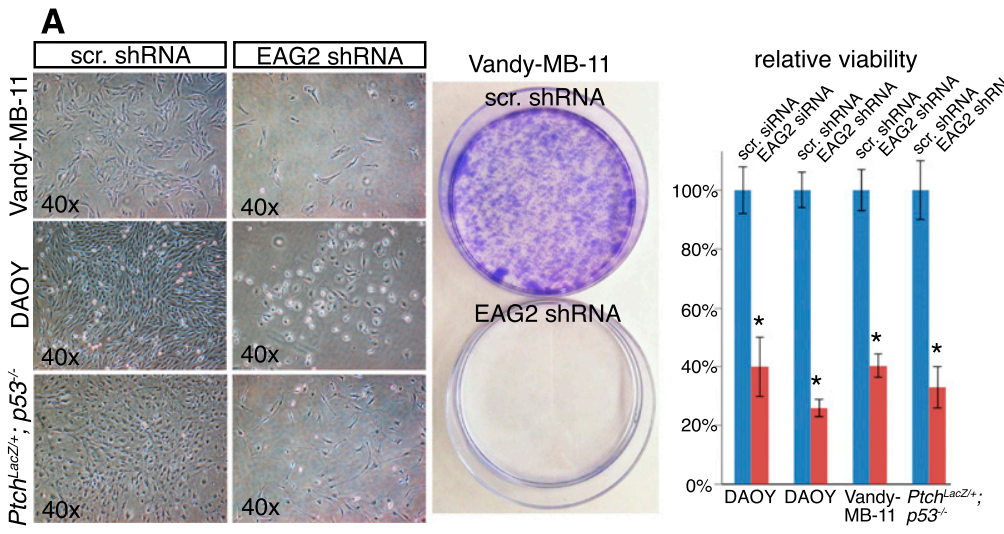

B

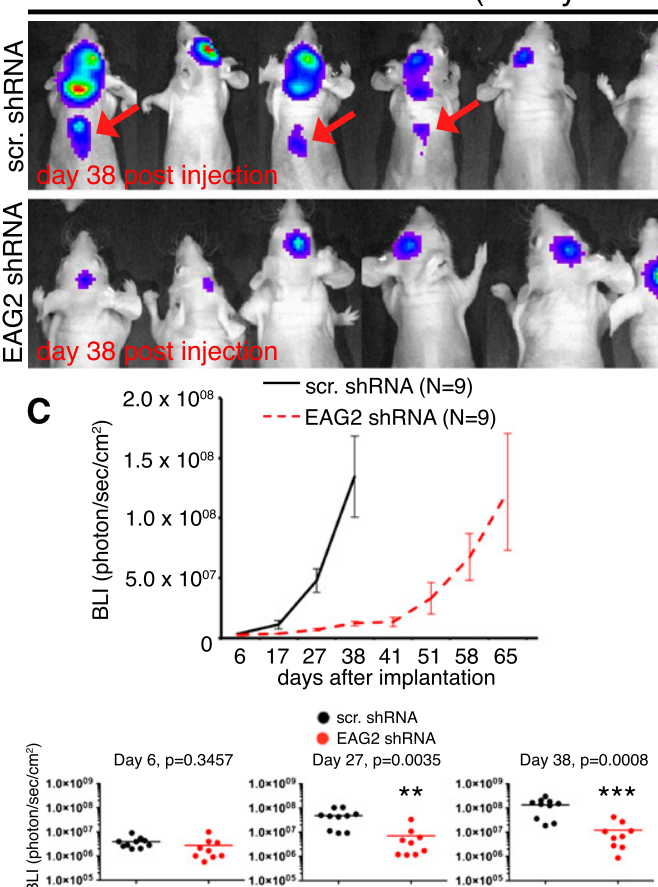

D

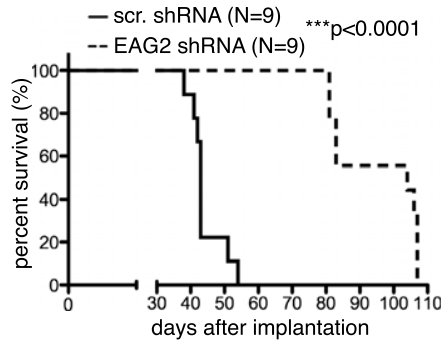

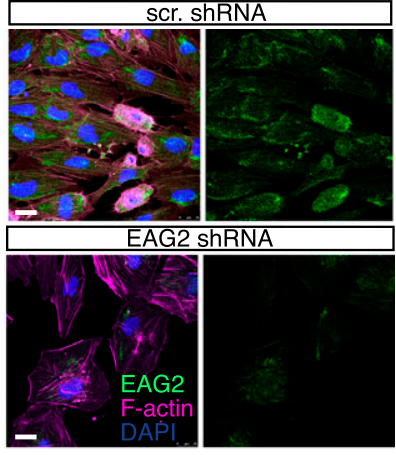

H.E.

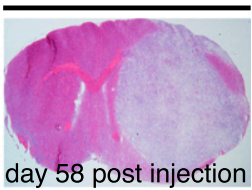

day 58 post injection

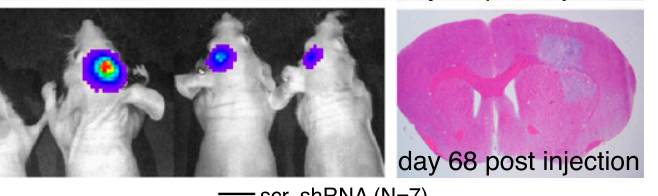

$\mathbf{E}$

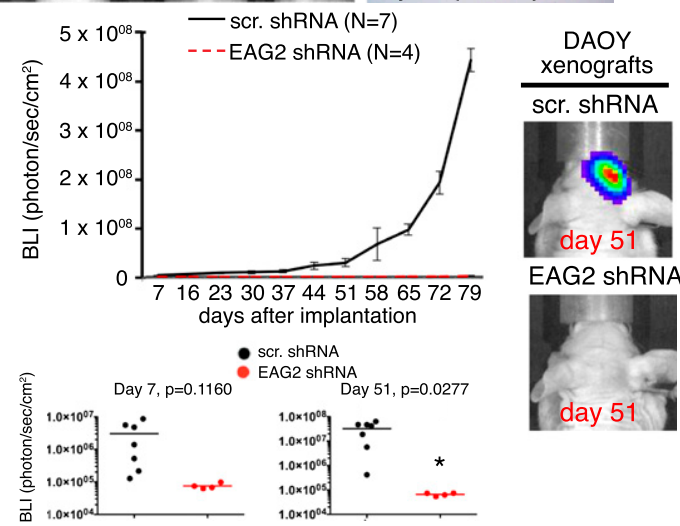

cr. $\operatorname{sh} R N A(N=7)$

$\mathbf{F}$

- scr. shRNA $(\mathrm{N}=7) \quad{ }^{* *} \mathrm{p}=0.0032$

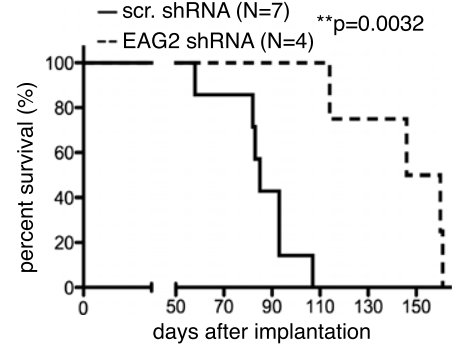

Figure 3. RNAi knockdown of EAG2 impairs MB cell growth and tumorigenicity. $(A)$ Representative images of comparably seeded DAOY cells, low-passage Vandy-MB-11 cells, and $\mathrm{Ptch}^{\mathrm{LacZ} /+} ; \mathrm{p} 53^{-/-}$mouse MB cells treated with scrambled/EAG2 shRNA for 7-10 d. The clonogenic capacity of EAG2 knockdown cells is dramatically impaired compared with the control cells. EAG2 RNAi knockdowns effectively reduce endogenous EAG2 protein expression. (B) Noninvasive bioluminescence imaging (BLI) shows significantly reduced tumor burden in the mice injected with EAG2-depleted Vandy-MB-11 cells. Note the presence of spinal cord metastases (red arrows) in the six out of nine $(67 \%)$ mice of only the control group. Histological analyses in another cohort of xenografted mice show that the tumors with EAG2 knockdown are much smaller in size. (C) While control tumors show steady growth rate, the EAG2 knockdown tumors display much slowed growth as measured by consecutive BLI measurements in Vandy-MB-11 xenografts. $(D)$ Kaplan-Meier survival analyses show that EAG2 knockdown in tumors significantly improves survival in the Vandy-MB-11 xenografted mice. $(E)$ While control tumors show a steady growth rate, the EAG2 knockdown tumors display much slower growth as measured by consecutive BLI measurements in the DAOY xenografts. $(F)$ Kaplan-Meier survival analyses show that EAG2 knockdown in tumors significantly improves survival in the DAOY xenografted mice. $\left(^{\star \star}\right) P<0.005 ;\left(^{\star}\right) P<0.05$ by Student's $t$-test. Bar, $A, 25 \mu \mathrm{m}$. 
in all of the recipient mice. In summary, these results demonstrate that EAG2 knockdown suppresses the tumorigenicity of $\mathrm{MB}$ cells in vivo, thereby improving overall survival of the tumor-bearing mice.

\section{EAG2 exhibits dynamic distribution in the $M B$ cell during different phases of the cell cycle}

To understand the mechanism by which EAG2 regulates $\mathrm{MB}$ cell growth, we first investigated the subcellular localization of EAG2 in dividing MB cells. While VandyMB-11 and DAOY cells exhibited little expression of EAG1 or HERG (Supplemental Fig. 7A), EAG2 was highly expressed in three different lines of low-passage human MB cells (established from resected tumors from patients Vandy-MB-6, Vandy-MB-10, and Vandy-MB-11) (Fig. 3A; Supplemental Fig. 7B; Supplemental Table 1) and DAOY cells (Supplemental Fig. 7C). Whereas EAG2 localized in intracellular punctate structures distant from the cell surface during interphase, distinguishable by the lack of chromosomal condensation (Fig. 4A; Supplemental Fig. $7 \mathrm{~B}, \mathrm{C})$, a portion of the EAG2 protein shuttled to the plasma membrane upon entering late G2 phase and remained on the cell surface during the course of mitosis (Fig. 4A; Supplemental Fig. 7C). Also, a high level of EAG2 expression was detected in the thin retraction fibers /white arrows in Fig. 4A) and cytoplasmic bridge (red arrow in Fig. 4A) during cytokinesis.

To further demonstrate the plasma membrane localization of EAG2 in mitotic MB cells, we transfected the Vandy-MB-11 cells with farnesylated eGFP. The 20-aminoacid farnesylation signal from $\mathrm{c}-\mathrm{Ha}$-Ras was fused to the $\mathrm{C}$ terminus of eGFP and directs farnesylated eGFP to the inner leaflet of the plasma membrane. We detected very robust temporal colocalization of EAG2 protein with the farnesylated eGFP specifically in mitotic MB cells, while the MB cells at interphase displayed largely intracellular EAG2 protein localization (Supplemental Fig. 8). These results further supported our observations that the distribution of EAG2 protein overlapped with that of the cortical F-actin associated with the cell membrane (Fig. 4A).

The dynamic shift in EAG2 membrane distribution was accompanied by changes in potassium current levels, as revealed by whole-cell voltage clamp recordings from DAOY cells at interphase and during mitosis. We determined the mitotic state of each cell by both cell morphology (mitotic cells are round, while cells in interphase tend to be flat) and DAPI staining (Fig. 4A,B). A slowly activating, outwardly rectifying, noninactivating current characteristic of EAG2 channels was the predominant current in mitotic DAOY cells (Fig. 4B). The amplitude of this current was more than twice as high in mitotic cells as cells at interphase (median current values $546 \mathrm{pA}$ vs. 202 pA at $+80 \mathrm{mV}$ ) (Fig. 4B), even though the mitotic cells were smaller in surface area (measured by cell capacitance) than the cells in interphase (Supplemental Fig. 9A). To account for this difference in cell size, we calculated the current density by dividing the current amplitude by the cell's capacitance. Mitotic cells had a fivefold increase in potassium current density compared with nondividing cells (median current density values 9.8 pA/pF vs. $2.1 \mathrm{pA} / \mathrm{pF}$ ) (Fig. 4B). This observation suggests that the selective membrane association of EAG2, in accordance with cell cycle status, elevates potassium channel activity during mitosis, raising the question of whether the EAG2 channel functions to promote mitotic entry and ensure successful mitosis in the MB cells.

\section{EAG2 knockdown results in $M B$ cell cycle arrest in the late G2 phase}

As EAG2 knockdown in MB cells led to severe MB cell growth defects as well as reduced tumor burden (Fig. 3; Supplemental Fig. 5), and EAG2 enrichment in cell membranes during mitosis was associated with increased potassium channel activity (Fig. 4A,B; Supplemental Fig. 8), we hypothesized that EAG2 is essential for MB cells to enter into and/or sustain mitosis. To test this, we conducted flow cytometry to determine the cell cycle profiles of Vandy-MB-11 cells treated with EAG2 shRNA or with scrambled shRNA as control. EAG2 knockdown caused a greater than twofold increase of cells in the G2/M phase $(271.8 \% \pm 20.2 \%$ vs. $100 \%$, after normalization with control MB cells in G2/M phase, $P<0.002$ ) (Fig. 4C), while the number of cells in G1 $(76.3 \% \pm 16.9 \%$ vs. $100 \%$ after normalization, $P>0.2$ ) (Fig. 4 C) or $S$ phase $(103.2 \% \pm$ $32.6 \%$ vs. $100 \%$ after normalization, $P>0.9$ ) (Fig. 4 C) was not significantly altered. We then performed immunostaining of phospho-histone 3 , whose weak or strong staining signal marks cells of late G2 and M phases, respectively. The cells of late G2 phase also displayed a lack of overtly condensed chromosomes (Fig. 4D). Consistent with the hypothesis that EAG2 is essential for mitotic entry, the phospho-histone 3-positive cells with EAG2 knockdown were predominantly in late G2 $(90.9 \%$ vs. $76.1 \%$ in control), so that the percentage of cells in each phase of mitosis was reduced $1.7 \%$ vs. $5.6 \%$ in control for prophase, $5.7 \%$ vs. $10.8 \%$ in control for prometaphase and metaphase, $1.7 \%$ vs. $7.5 \%$ in control for anaphase and telophase, three independent knockdown experiments with a total of 536 cells with EAG2 knockdown and 418 control cells counted). We found a similar defect in G2/M cell cycle progression of DAOY cells with EAG2 knockdown (data not shown). Taken together, these findings indicate that EAG2 is essential for mitotic entry of $\mathrm{MB}$ cells.

\section{MB cells with EAG2 knockdown display aberrant premitotic cytoplasmic condensation (PMC) and mitotic catastrophe}

To further study how EAG2 regulates mitosis, we performed time-lapse phase-contrast microscopy to monitor the entire process from mitotic entry to cytokinesis. The initiation of cell volume reduction during PMC marks the onset of mitosis (Habela and Sontheimer 2007; Boucrot and Kirchhausen 2008), which we define as time 0. Strikingly, while mitotic progression proceeded normally in most of the control MB cells (33 of 34 successfully completed cytokinesis with two daughter cells, 97\%), with an average mitotic duration of $161.2 \pm 28.5 \mathrm{~min}$ (Fig. 
A

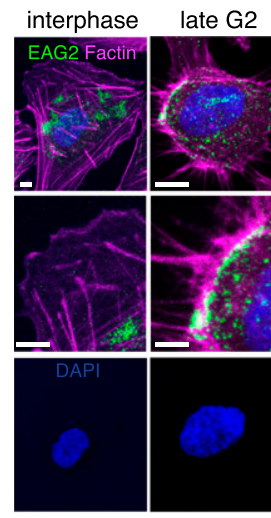

C

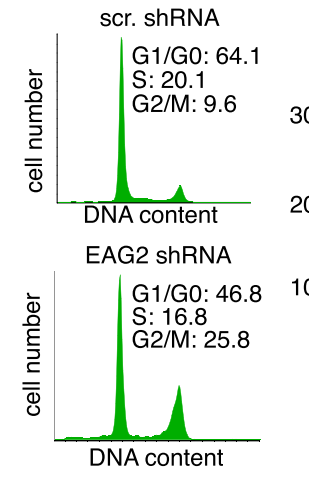

$\mathbf{E}$

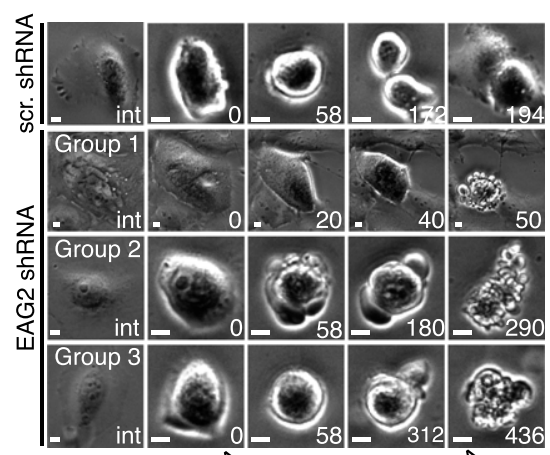

H

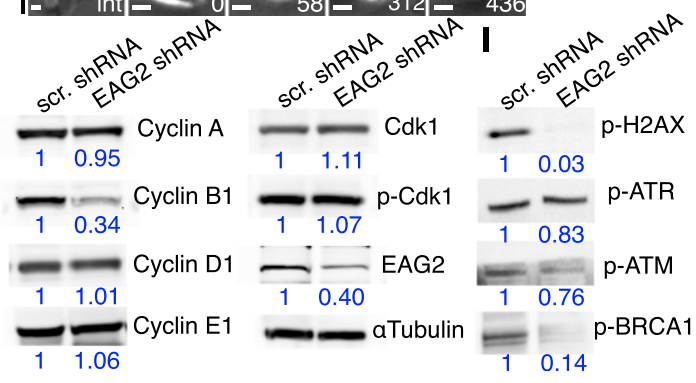

B
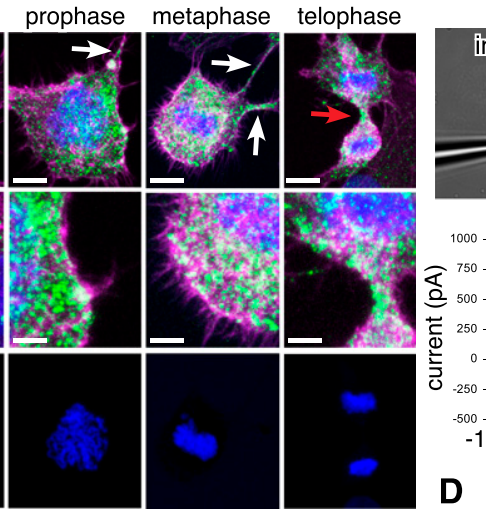

F
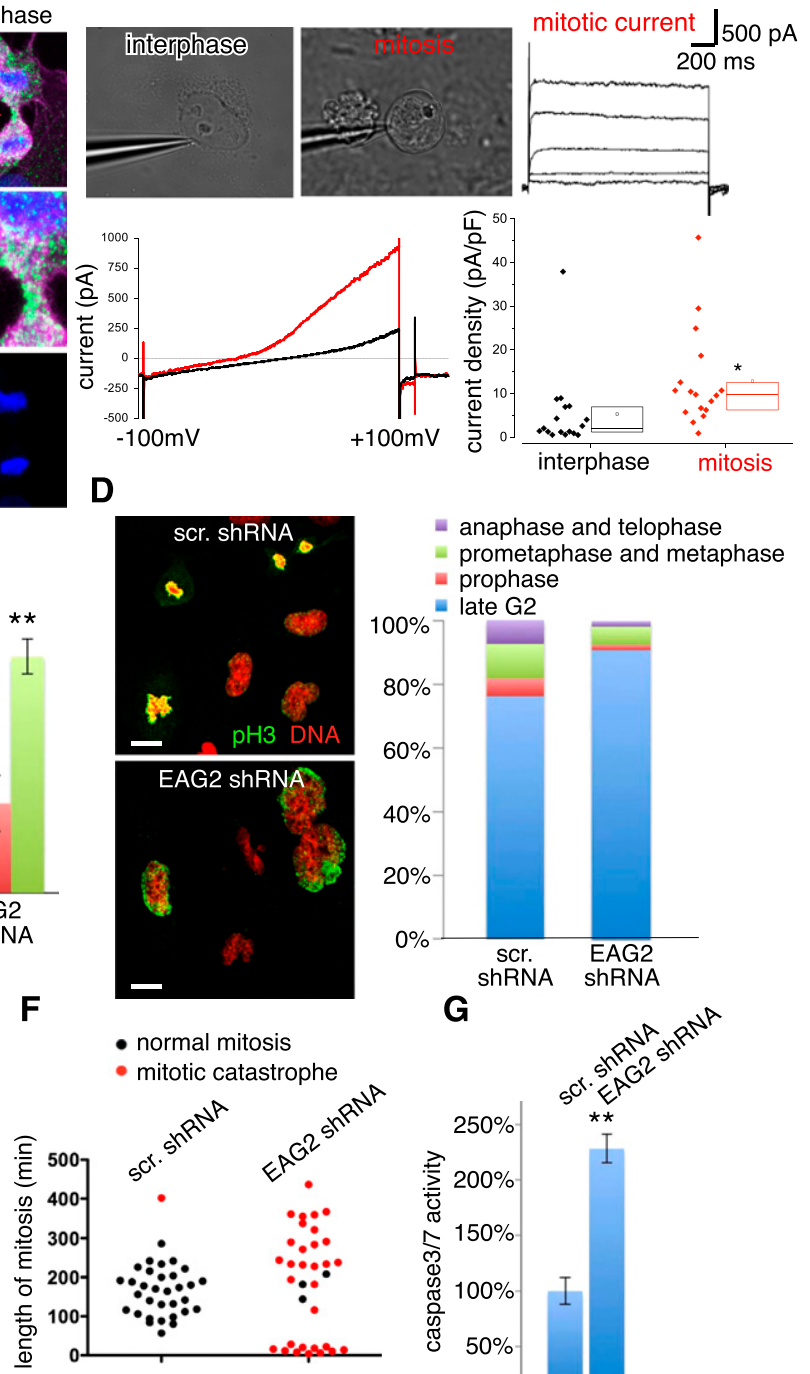

$$
\begin{aligned}
& \text { - anaphase and telophase } \\
& \text { " prometaphase and metaphase } \\
& =\text { prophase }
\end{aligned}
$$
$100 \%$ late G2

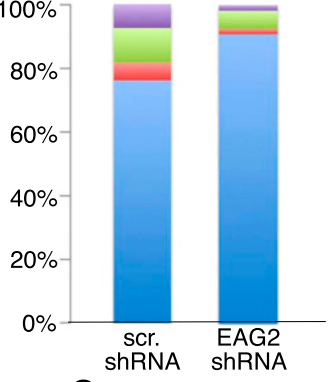

G
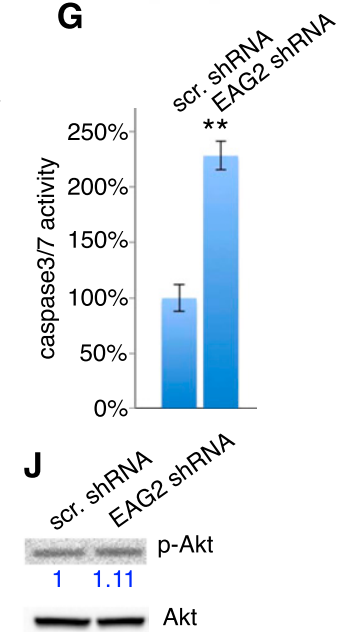

Figure 4. Dynamic EAG2 protein localization and profound mitotic phenotype associated with EAG2 knockdown. $(A)$ EAG2 localizes within the intracellular compartments of the Vandy-MB-11 cells during interphase, as evidenced by the lack of immunostaining signal in the cell periphery. EAG2 protein can be detected on the cell surface, outlined by cortical F-actin in Vandy-MB-11 cells at late G2 phase and mitosis. $(B)$ The top left panels show representative images of whole-cell recording from DAOY cells during interphase and mitosis. The top right traces show representative whole-cell voltage clamp traces from mitotic DAOY cells, revealing a prominent slowly activating outward current. Cells were voltage-clamped at a holding potential of $-80 \mathrm{mV}$, then stepped from $-60 \mathrm{mV}$ to +100 $\mathrm{mV}$ in 40-mV steps. Dividing DAOY cells display significantly greater outward potassium current both in absolute amplitude (bottom left) and in terms of current density (bottom right). $\left(^{\star}\right) P<0.01$ by Mann-Whitney $U$-test. (C) FACS analyses of Vandy-MB-11 cells treated with control and EAG2 shRNA show a significant increase in the proportion of cells in the G2/M phase following EAG2 knockdown. (D) Phospho-histone 3 staining shows significantly fewer cells with strong immunopositivity on condensed chromosomes and more cells with weak, diffuse nuclear immunopositivity after EAG2 knockdown. (E) Time-lapse microscopy on Vandy-MB-11 cell mitosis (in minutes). (F) Quantification of mitotic duration. $(G)$ Relative Caspase 3/7 activity in Vandy-MB-11 cells. $(H)$ Western blotting shows reduced EAG2 and Cyclin B1 expression after EAG2 knockdown, while Cyclin A, Cyclin D1, Cyclin E1, Cdk1, and phospho-Cdk1 expression is comparable between knockdown and controls. $(I)$ Western blot showing decreased DNA damage and DNA damage response (DDR) pathway activation and comparable p53 phosphorylation in the EAG2-depleted Vandy-MB-11 cells compared with the control cells. $(J)$ Western blot showing comparable level of phospho-AKT protein in knockdown and control cells. Bars: $A, E, 10 \mu \mathrm{m} ; D, 20 \mu \mathrm{m}$. 
4E,F), those MB cells with EAG2 knockdown that initiated mitotic entry displayed profoundly defective mitotic morphologies and eventually underwent apoptosis through mitotic catastrophe.

We observed three distinct patterns of defective mitotic entry. The first group of EAG2-depleted MB cells initiated PMC but never reached metaphase or maximal mitotic rounding. These cells rapidly encountered mitotic catastrophe with much shortened mitotic duration (14.3 \pm 3.7 min, 11 of 11 died, 100\%) (Fig. 4E,F). The second group of cells achieved a near-complete rounded morphology but with many abnormal cellular protrusions and blebbings, along with the appearance of a nonuniform membrane. The third group of cells initially completed mitotic rounding but failed to maintain it. The two latter groups of EAG2-depleted cells rarely exited mitosis and often died after prolonged mitotic block $(265.9 \pm 40.1 \mathrm{~min}, 20$ of 23 died, 87\%) (Fig. 4E,F). Consistent with these profound apoptotic processes, we detected significantly increased Caspase 3 and Caspase 7 activity in the MB cells with EAG2 knockdown (Fig. 4G).

To further confirm the mitotic defect at the molecular level, we assessed the expression of a panel of Cyclin genes that are central for cell cycle progression. In line with the cellular phenotypes, we found a strong and selective decrease of Cyclin B1 that is essential for G2/ $M$ transition (Fig. 4H), while the expression of Cyclin A, Cyclin D1, Cyclin E1, Cdk1, and phosphorylated Cdk1 was not significantly altered by EAG2 knockdown (Fig. 4H). Thus, EAG2 knockdown led to significant G2 arrest and robust mitotic catastrophe associated with abnormal PMC and mitotic morphology, supporting the notion that the membrane localization of EAG2 and enhanced outward potassium current are essential for mitotic entry and successful mitosis.

The tumor suppression function of EAG2 knockdown is independent of p53 activation/expression, DNA damage checkpoint activation, or changes in the AKT pathway

To study the molecular mechanisms underlying the EAG2 regulation of $\mathrm{MB}$ cell growth, we first set out to assess the impact of EAG2 knockdown on the DNA damage response (DDR) pathway. While activation of the DDR pathway is known to elicit G2 arrest and apoptosis (Zhou and Elledge 2000), we found that EAG2 knockdown resulted in a reduced rather than increased activation of this pathway, as evidenced by markedly decreased phosphorylation of H2AX in the EAG2-depleted MB cells. In line with the decreased expression of phospho-H2AX, the activation/phosphorylation of a battery of DDR pathway proteins was reduced compared with control cells (Fig. 4I). It seems likely that the prolonged G2 arrest may have allowed more time for the MB cells to repair the DNA damage from replication errors during their normally rapid cell divisions, as reported previously (Liu et al. 2011). In agreement with our finding that EAG2 knockdown also resulted in a robust growth defect in the Ptch ${ }^{\text {LacZ/+ }} ;$ p53 ${ }^{-/-}$mouse MB cells (Fig. 3A), we detected no overt change in the phosphorylation level of p53 in Vandy-MB-11 cells (Fig. 4I), indicating that p53 expression or pathway activation was also not required for suppression of cell growth by EAG2 knockdown. Furthermore, while activation of the PI3K/AKT pathway has been shown to promote MB growth (Wlodarski et al. 2006; Yang et al. 2010; Pei et al. 2012), the levels of phosphorylated AKT in control and EAG2-depleted MB cells were comparable (Fig. 4J), suggesting that EAG2 does not engage the AKT pathway to regulate $\mathrm{MB}$ cell growth.

\section{EAG2 knockdown results in a striking increase in cell volume in late G2 phase}

Mammalian cells undergo dynamic cell volume changes during cell cycle progression. Both adherent and nonadherent cells decrease their volume just before entering mitosis through $\mathrm{PMC}$, reaching a minimal volume at metaphase while completing mitotic rounding, followed by increased volume at cytokinesis (Habela and Sontheimer 2007; Boucrot and Kirchhausen 2008). Since we observed a temporal correlation of EAG2 translocation to the cell membrane with mitotic entry from the late G2 phase concurrent with an increase of outward potassium current during mitosis, the defects of EAG2depleted cells in achieving and maintaining proper mitotic morphology prompted us to hypothesize that EAG2 functions to regulate cell volume during the MB cell cycle progression. In line with the reported cell volume dynamics (Habela and Sontheimer 2007; Boucrot and Kirchhausen 2008), control Vandy-MB-11 cells displayed larger cell volumes at interphase $\left(6148.8 \pm 1111.6 \mu \mathrm{M}^{3}\right.$, $N=11$ ) and late G2 phase (marked by punctate phosphohistone 3 immunoreactivity and noncondensed chromosomes, $7600.2 \pm 1265.6 \mu \mathrm{M}^{3}, N=24$ ) (Fig. 5A) and reduced cell volumes during mitosis (4368.7 \pm 579.6 $\mu \mathrm{M}^{3}, N=8$ at prophase, $1879.3 \pm 151.2 \mu \mathrm{M}^{3}, N=16$ at prometaphase and metaphase, $1533.2 \pm 182.8 \mu \mathrm{M}^{3}, N=8$ at anaphase and telophase) (Fig. 5A). In contrast, $\mathrm{MB}$ cells with EAG2 knockdown had a much larger cell volume in the late G2 $\left(20971.8 \pm 5751 \mu \mathrm{M}^{3}, N=14, P<3.04 \times 10^{-6}\right)$ (Fig. 5A) than those of control cells, while their volumes at interphase were within the normal range (7208.2 \pm $1743.7 \mu^{3}, N=21, P>0.36$ ) (Fig. 5A). This abnormal increase in cell volume at late G2 phase effectively abrogated PMC and hence may have triggered a volume checkpoint control to prevent mitotic entry. Interestingly, there was no difference between the cell volumes for the EAG2-depleted MB cells that did enter mitosis (4246.3 $\pm 608.5 \mu \mathrm{M}^{3}, N=8$ at prophase, $P>0.83$; $1863.7 \pm$ $254.1 \mu \mathrm{M}^{3}, N=13$ at prometaphase and metaphase, $P>$ $0.91 ; 1711.2 \pm 128.4 \mu \mathrm{M}^{3}, N=8$ at anaphase and telophase, $P>0.29$ ) (Fig. 5A) and those of control cells. These results suggest that there is a stringent cell volume checkpoint that the $\mathrm{MB}$ cells need to pass for mitotic entry. As a consequence of their inability to reduce cell volume properly, those cells with EAG2 knockdown that manage to escape this checkpoint and enter mitosis may eventually encounter mitotic catastrophe (Fig. 4E,F). 
A

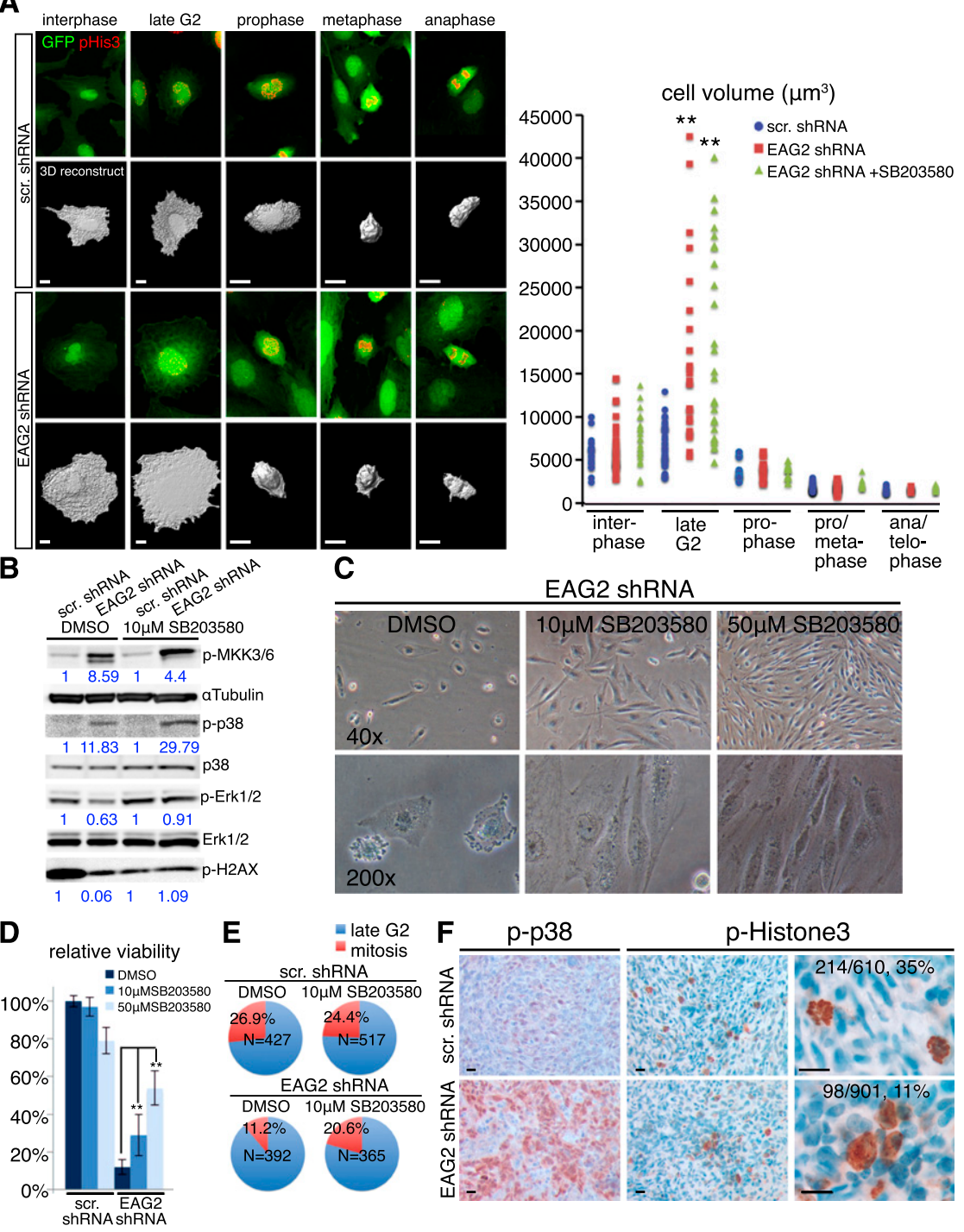

Figure 5. EAG2 knockdown results in defective PMC, with cell volume increase at late G2 phase, associated with activation of the tumor suppressor p38 MAPK pathway. (A) Cell volume measurements of soluble GFP-filled Vandy-MB-11 cells. GFP expression in Vandy-MB-11 cells is achieved by infection with GFP-expressing lentivirus. (B) EAG2-depleted cells display robust activation of the p38 MAPK pathway. The ERK pathway is modestly regulated by EAG2 function and p38 inhibition. SB203580 normalizes the DNA damage in EAG2-depleted MB cells. $(C, D)$ The p38 MAPK inhibitor SB203580 rescues EAG2-depleted Vandy-MB-11 cell growth phenotype in a dose-dependent manner. (E) SB203580 promotes G2-M transition after EAG2 knockdown. $(F)$ Following EAG2 knockdown, Vandy-MB-11 xenografted tumors display hyperactivation of the p38 MAPK pathway with prominent phospho-p38 staining and a significantly decreased percentage of cells stained with phospho-histone 3 and with condensed chromosomes, indicating defective mitotic entry in vivo $(N$ $=3$ each for control and EAG2 knockdown tumors). Bars: $A, 20 \mu \mathrm{m} ; F, 15 \mu \mathrm{m}$.

\section{EAG2 knockdown results in p38 MAPK pathway activation that blocks $M B$ cell proliferation}

Next we investigated how the increased cell volume of cells with EAG2 knockdown causes G2 arrest. The p38 MAPK pathway, previously shown to negatively regulate malignant cell growth and tumorigenesis by inducing G2-M cell cycle arrest and apoptosis (Han and Sun 2007), can be activated by various external stimuli such as bacterial infection, inflammatory cytokines, and UV radiation. Because osmotic shock also potently induces p38 MAPK activation through dual tyrosine/threonine phosphorylation mediated by two upstream MAPK kinases (MEKs), MEK3 and MEK6 (Marshall et al. 2005), we wondered whether the cell volume increase in the EAG2depleted cells, which mimics the cellular outcome of hypotonic stress, would lead to p38 MAPK pathway activation, thereby causing G2 cell cycle arrest and suppression of tumor growth.

Indeed, EAG2 knockdown led to a robust increase in the phosphorylation of p38 at Thr 180 and Tyr 182 as well as phosphorylation of the upstream kinases MEK3 and MEK6 (Fig. 5B). To test whether the p38 MAPK pathway lies downstream from the cell volume change to suppress $\mathrm{MB}$ cell growth, we asked whether directly inhibiting $\mathrm{p} 38$ MAPK activity would rescue the cell growth despite increased cell volume due to EAG2 knockdown. Indeed, treatment with the small molecule p38 inhibitor SB203580 significantly rescued the growth of EAG2-depleted MB cells in a dose-dependent manner (Fig. 5C,D). SB203580 inhibits 38 MAPK catalytic activity by binding to the ATPbinding pocket but does not reduce the phosphorylation of p38 MAPK by upstream kinases (Fig. 5B; Kumar et al. 1999). Importantly, these SB203580-treated MB cells exhibited normalized levels of DNA damage and an increased G2-M transition rate (Fig. 5B,E) while still displaying significantly increased cell volumes as compared with control cells (Fig. 5A), suggesting that the cell volume decrease of PMC prior to mitosis is detected by a checkpoint that can be triggered in cells not achieving PMC to activate the $\mathrm{p} 38$ pathway and prevent mitotic entry. The 
reduced level of phospho-H2AX in control cells with SB203580 compared with DMSO treatment is consistent with the reported function of $\mathrm{p} 38$ to directly phosphorylate $\mathrm{H} 2 \mathrm{AX}$ (Lu et al. 2008). The p42/p44 ERK pathway was only modestly affected by EAG2 knockdown and changes to p38 pathway activity (Fig. 5B), consistent with the primary and selective engagement of the p38 pathway in cells with EAG2 knockdown. Furthermore, we detected hyperactivation of the p38 MAPK pathway and a strong G2-M transition defect in xenografted VandyMB-11 tumors with EAG2 knockdown (Fig. 5F). Taken together, these findings suggest that EAG2 is essential for dynamic cell volume control during MB cell cycle progression and establish the p38 MAPK pathway as a tumor suppressor pathway downstream from EAG2 knockdown to reduce MB tumor growth.

Ectopic EAG2 expression leads to strong cell volume reduction and cell cycle defects

As EAG2 critically regulated MB cell growth, and EAG2 knockdown induced pronounced tumor suppression phenotypes, we wondered whether overexpressing EAG2 in nontumor cells would impact cellular proliferation and overall growth. Remarkably, we found that overexpressing EAG2 in COS7 and HEK293 cells via transfection significantly reduced cell volumes and altered their morphology $\left(5028 \pm 333.6 \mu \mathrm{M}^{3}, N=30\right.$ for GFP-expressing COS7 cells vs. $2026 \pm 248.2 \mu \mathrm{M}^{3}, N=30$ for EAG2overexpressing COS7 cells, $P<0.0001 ; 3487 \pm 217.1 \mu \mathrm{M}^{3}$, $N=30$ for GFP-expressing HEK293 cells vs. $1395 \pm 72.01$ $\mu \mathrm{M}^{3}, N=30$ for EAG2-overexpressing HEK293 cells, $P<$ 0.0001) (Fig. 6A). EAG2 overexpression often resulted in the formation of many ectopic retraction fibers, reminiscent of those seen during mitosis (Figs. 4A, 6A,C). EAG2 overexpression also often caused the cells to lose their flattened, spread-out morphology during interphase and instead exhibit near-rounded cellular morphologies resembling the normal mitotic rounding phenomenon, even in the absence of detectable phospho-histone 3 and chromosomal condensation, indicating that the transfected cells were still at interphase (Fig. 6A,C). In agreement with the importance of cell volume oscillations during normal cell cycle progression, we found that the persistent cell volume reduction actually resulted in significantly impaired cell growth with defective cell cycle progression and increased apoptotic cell death (Fig. 6B). Notably, while endogenous EAG2 normally localized intracellularly in MB cells at interphase (Fig. 4A; Supplemental Fig. 8), exogenously overexpressed EAG2 protein was clearly detected on the plasma membrane demarcated by farnesylated eGFP of the transfected COS7 cells at interphase (Fig. 6C), further suggesting that the cell surface localization of EAG2 is essential for its function in regulating cell volume dynamics. As a control, overexpressing Kv4.2, another voltage-gated potassium channel conducting transient outward current with rapid inactivation, did not elicit overt cell volume changes or an apparent growth phenotype (Fig. 6A,B). In summary, these results suggest that the temporally regulated plasma membrane localization of EAG2 is essential for its function to regulate cell volume changes during cell cycle progression and successful mitosis and that the upregulation of EAG2 expression in $\mathrm{MB}$ cells provides a sufficient total EAG2 protein repertoire to accommodate rapid cell proliferation and its membrane function when needed.

\section{Discussion}

It is highly desirable to identify specific molecules critical for tumor growth and progression for the future development of targeted therapies. Our study is the first exploration of the role of an ion channel in the tumorigenesis of $\mathrm{MB}$ in vitro and in vivo. Our genome-wide microarray analysis identified the voltage-gated potassium channel Eag2 as the most up-regulated ion channel gene in Shhdriven mouse MBs. Our molecular and electrophysiological studies verified the abundance of Eag2 protein and potassium channel activity in $\mathrm{MB}$ cells. We further established that EAG2 overexpression is a hallmark of a significant subset of human MBs across three of the four molecularly defined subgroups. Our functional studies involving EAG2 knockdown demonstrated the essential roles of EAG2 during MB cell growth in vitro and tumorigenicity in vivo. Mechanistically, we uncovered an unprecedented dynamic shuttling of EAG2 during different phases of the cell cycle, leading to a higher outward potassium current density during mitosis. To test for the functional importance of EAG2 for mitotic entry and the maintenance of mitosis as suggested by the EAG2 enrichment on the cell membrane during mitosis, we examined MB cells with EAG2 knockdown and elucidated the basis for the resulting impairment of mitotic entry associated with reduced Cyclin B1 expression by showing that EAG2 is an essential regulator for the cell volume reduction during PMC of MB cells. Reducing EAG2 expression hampers the ability of $\mathrm{MB}$ cells to condense their volume and enter mitosis, triggering a volume checkpoint control to induce robust p38 MAPK tumor suppressor pathway activities to inhibit tumor growth. Conversely, ectopic membrane EAG2 function leads to marked cell volume reduction and mitotic-like morphology in cells at interphase and impaired growth with cell cycle defects and apoptosis (Fig. 7). The EAG2 overexpression across several histological and molecular subgroups highlights its potential therapeutic value as a target for drug development in a substantial subset of MBs. As genomic amplifications of several other potassium channels, including KCNB2, KCNI1, KCNI2, KCNK16, $K C N E 1$, and KCNE2, have been previously reported in human MBs (Northcott et al. 2009), our study also provides a strong impetus for studying the efficacy of targeting ion channels to treat this devastating brain tumor.

Activation of the p38 MAPK pathway can lead to cellular senescence, growth arrest, or differentiation, thereby allowing more time for the cell to cope with damaging external stimuli such as inflammation, UV radiation, oncogenic stress, and osmotic shock or induce apoptosis to eliminate cells at risk (Han and Sun 2007; Wagner and Nebreda 
A
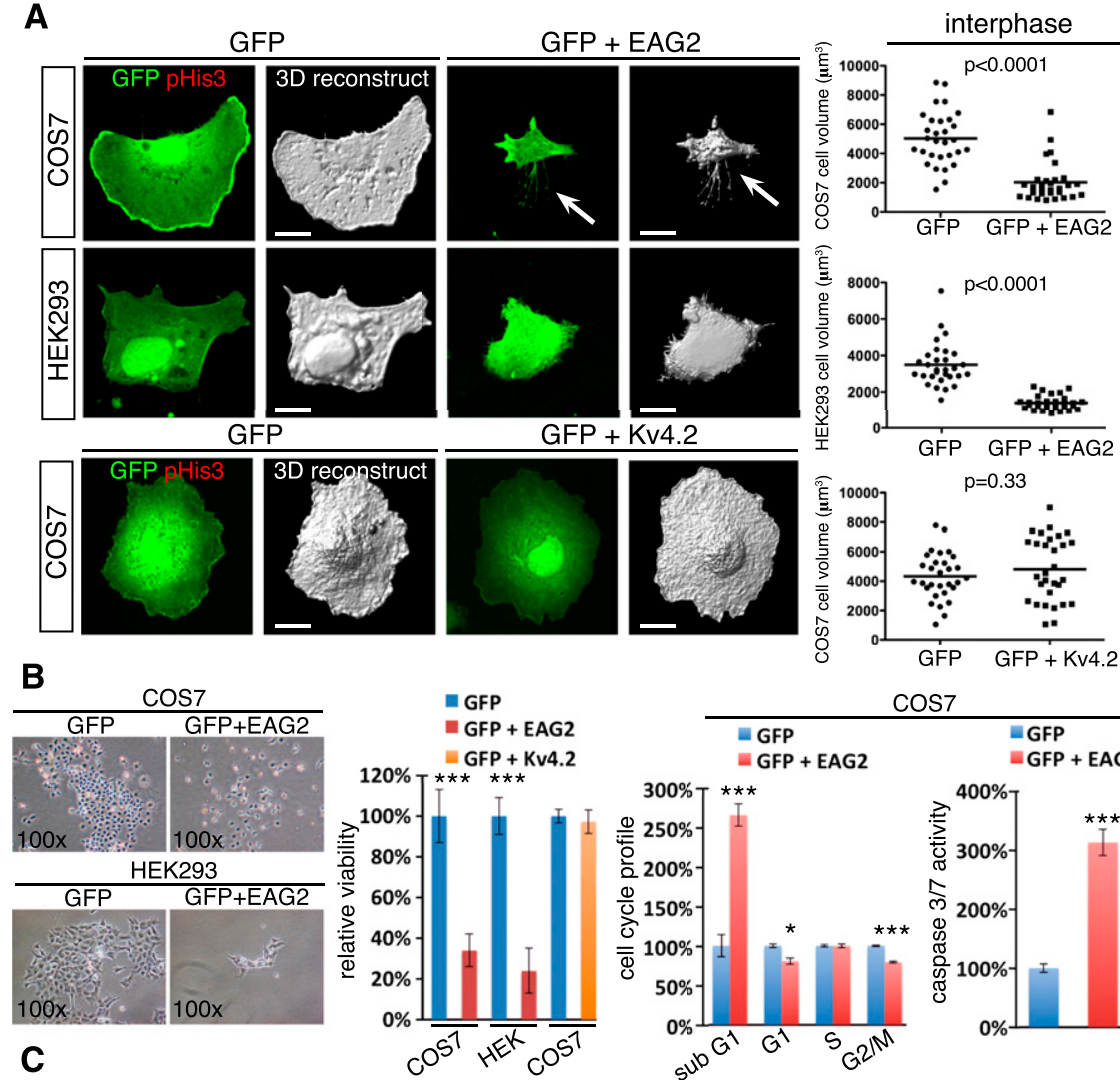

\section{C}
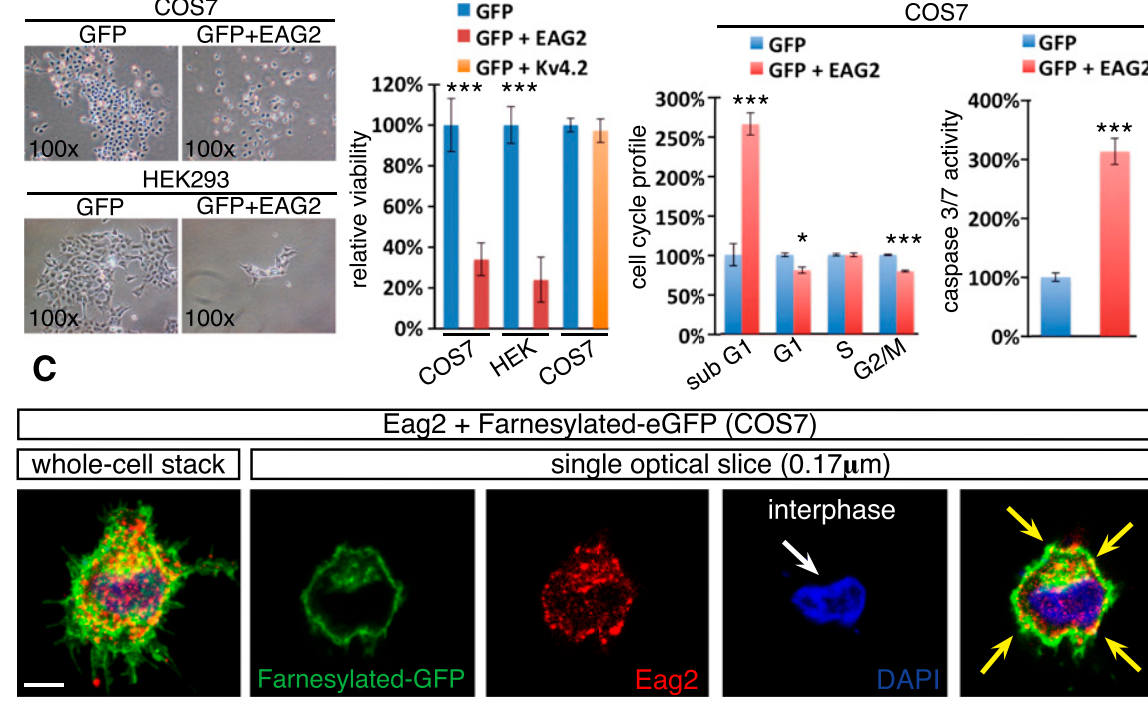

Eag2 + Farnesylated-eGFP (COS7) single optical slice $(0.17 \mu \mathrm{m})$
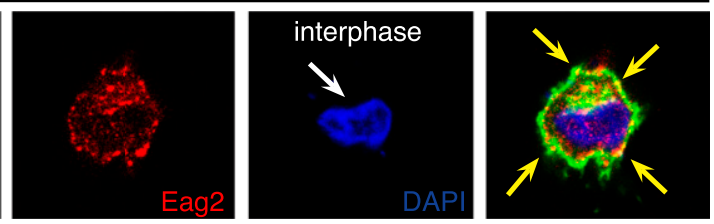

Figure 6. Ectopic EAG2 expression leads to cell volume reduction and cell cycle defects. $(A)$ Cell volume measurements of soluble GFP-filled COS7 and HEK293 cells. COS7 and HEK293 cells are transfected with GFP, GFP + EAG2, or GFP + Kv4.2. Overexpression of EAG2, but not Kv4.2, strongly reduces COS7 and HEK293 cell volumes. The COS7 and HEK293 cells are at interphase, as evidenced by their lack of phospho-histone 3 immunoreactivity. Note that prominent ectopic retraction fibers are seen only in EAG2 transfected cells (white arrow). (B) Overexpression of EAG2, but not Kv4.2, leads to a profound growth phenotype with defective cell cycle and apoptosis. White-field images of comparably seeded COS7 and HEK293 show that EAG2 overexpression reduces the overall cell growth. EAG2 overexpression leads to a significant reduction of cells at G1 and G2/M phases as well as a marked increase of apoptotic cell death, as evidenced by the increase of cells at the sub-G1 phase and Caspase 3/7 activity. $(C)$ Transfected EAG2 localizes to the plasma membrane marked by farnesylated eGFP. Both the whole-cell three-dimensional stacked and single optical slice views of a typical EAG2-overexpressing COS7 cell are shown. Note the prominent plasma membrane localization of EAG2 (yellow arrows). Bars: $A, 20 \mu \mathrm{m} ; B, 10 \mu \mathrm{m}$.

2009). However, in some circumstances, p38 MAPK pathway activation has been found to promote cell proliferation (Hegle et al. 2006; Ayllon and O'Connor 2007), as is the case for CGNPs (Guldal et al. 2012). In our study, the p38 MAPK inhibitor SB203580 had minimal effects on Vandy-MB-11 cell growth but profoundly reversed the growth defects of EAG2-depleted cells (Fig. 5C,D). This suggests that the p38 MAPK pathway is not essential for Vandy-MB-11 cell growth and may be a potent tumor suppressor pathway induced by EAG2 deficiency. EAG2 knockdown induced a very robust (11.83-fold) increase in the phospho-p38 level. It is possible that this level of p38
MAPK pathway activation exceeded that of the CGNPs treated with Shh (Guldal et al. 2012) so that it prevented cell proliferation. It is also possible that the p38 MAPK pathway is protective for CGNP growth by preventing excessive oxidative and genotoxic stress associated with rapid cell division. Finally, the p38 MAPK pathway may regulate proliferation and survival differently in CGNP and $\mathrm{MB}$ cells. The full spectrum of potentially MB subgroupspecific roles of p38 MAPK requires further investigation.

Ion channels are present in essentially all cells and regulate membrane potential and electrical signaling in excitable cells (Hille 2001). Aberrant ion channel functions 
A

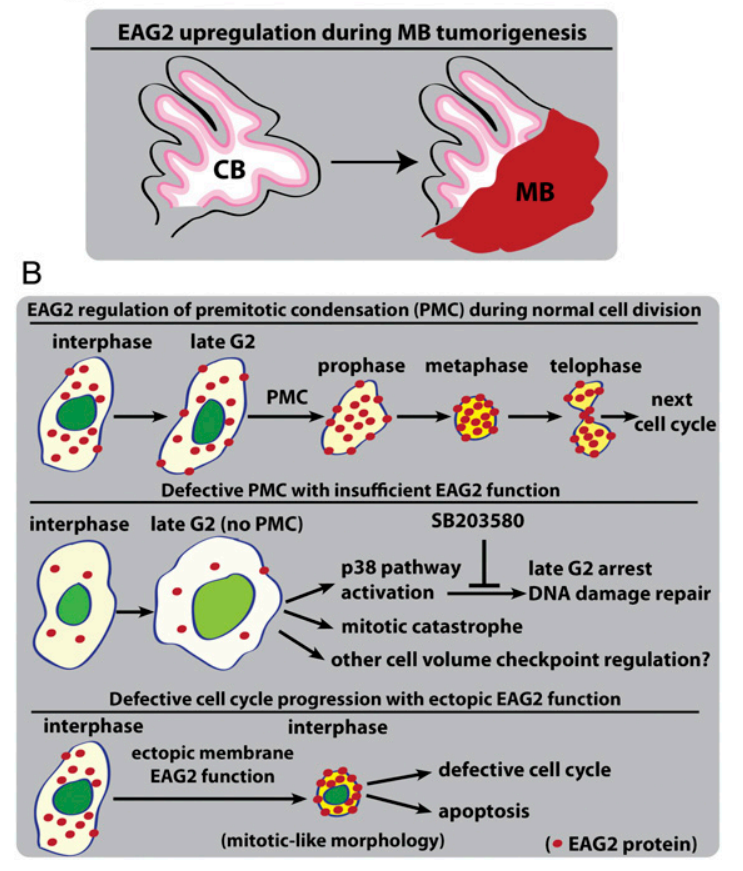

Figure 7. Model of EAG2's regulation on MB growth. (A) EAG2 protein is normally expressed at modest levels in the adult cerebellum and is significantly up-regulated in a subset of MBs. (B) EAG2 confers a growth advantage to $\mathrm{MB}$ cells by promoting cell cycle progression through the regulation of PMC, the abrogation of which leads to strong mitotic defects associated with hyperactivation of the p38 MAPK tumor suppressor pathway. Ectopic membrane expression of EAG2 in nontumor cells results in strong cell volume reduction, the formation of a mitotic-like morphology, defective cell cycle progression, and apoptosis.

contribute to the pathogenic conditions such as epilepsy, cardiac arrhythmia, and neuromuscular symptoms collectively known as channelopathies (Ashcroft 2000). Increasing evidence suggests that cancer can be considered another category of disease in the channelopathies, since up-regulated expression of several classes of ion channels, including potassium channels, sodium channels, calcium channels, transient receptor potential channels, and chloride channels, has been found in various types of cancers, and the application of ion channel blockers can inhibit cancer cell growth (Pardo 2004; Fraser and Pardo 2008; Prevarskaya et al. 2010). Several potassium channels have been implicated in cancer. For example, the two-pore potassium channel TASK-3 (KCNK9) gene amplification has been detected in breast, lung, and colorectal cancers, and its ectopic expression enhances tumorigenicity in nontransformed cells (Mu et al. 2003; Kim et al. 2004). Selective up-regulation of HERG in several human neoplasms, but not their normal tissue counterparts, has been reported, and pharmacological inhibition of the HERG channel is efficacious in reducing tumor cell proliferation in primary leukemic cells (Pillozzi et al. 2002; Jehle et al. 2011). Aberrant EAG1 expression has been reported in $>75 \%$ of human tumors, and its inhibition decreases the proliferation of EAG1-expressing cells (Pardo et al. 2005; Hemmerlein et al. 2006; Mello de Queiroz et al. 2006). While potassium channels have been implicated in cancer, little is known about how ion channels regulate tumor growth and progression. EAG2 is the second closest ortholog of the Drosophila eag gene and shares similar electrophysiological properties with EAG1. Prior to this study, EAG2 had not been implicated in either cell proliferation or cancer growth and remained essentially uncharacterized with respect to any physiological or pathological role. Our study represents the first molecular characterization of $\mathrm{MB}$ as a channelopathy. Whereas the expression levels of potassium channels previously implicated in cancer were either unchanged or down-regulated in the Shh-driven mouse MBs, we found that Eag2 was uniquely overexpressed. While research on the contributions of ion channels in the initiation, maintenance, and progression of cancer is in the nascent stages, drugs targeting ion channels represent a potentially promising therapeutic avenue for potential treatment of cancers owing to their highly druggable nature and their accessible cell surface localization. With the overexpression and heightened requirement of EAG2 function in MB cells, future investigations are warranted to determine whether existing EAG2 blockers can be used to selectively target neoplastic brain tissues with tolerable side effects or whether novel EAG2-blocking agents with greater potency and selectivity need to be developed. The fact that EAG2 overexpression was found in MBs of different subgroups and the fact that no EAG2 mutation has been detected in the previously profiled exomes of $22 \mathrm{MBs}$ (Parsons et al. 2011) also raise the intriguing possibility that targeting the wild-type EAG2 protein may provide a valid avenue for personalized medicine for a spectrum of MB patients.

Potassium channels may use several mechanisms to regulate cell proliferation. While cancer cells generally maintain moderately depolarized membrane potential compared with nontransformed cells, transient hyperpolarization has been reported to be necessary for successful G1-S cell cycle progression (Wonderlin and Strobl 1996). Therefore, it is possible that cancer cells increase the expression of specific potassium channels as a regulatory mechanism for temporally regulated hyperpolarization during cell division. As such, ion channel expression and function may vary depending on the cell cycle phase. For example, a previous study demonstrated that EAG1 expression is dependent on the cell cycle in human neuroblastoma cells (Meyer and Heinemann 1998), and a heterologous expression study in Xenopus oocytes has demonstrated changes in the electrophysiological properties of rat Eag1 that depend on the mitosis-promoting factor (Pardo et al. 1998). Similarly, the expression and voltage dependence of HERG channel activation differ during the cell cycle, which may reflect another venue for dynamic regulation of cell proliferation (Arcangeli et al. 1995; Crociani et al. 2003). Potassium channels, however, may also promote cell proliferation independent of ion permeation functions. It has been shown that transfecting NIH3T3 fibroblasts and C2C12 myoblasts with either wild-type EAG1 or a mutant form of EAG1 that cannot 
conduct current promotes proliferation (Hegle et al. 2006), and ectopically expressing wild-type or pore-dead mutant EAG1 in CHO cells promotes the growth of xenografts with enhanced vascularization to similar extents (Downie et al. 2008). Indeed, the intracellular domains of potassium channels can interact with signaling molecules to directly regulate cellular behaviors, as exemplified by the association of EAG1 with calmodulin (Schonherr et al. 2000; Ziechner et al. 2006; Goncalves and Stuhmer 2010) and the association of HERG with Src tyrosine kinases (Cayabyab and Schlichter 2002).

As a stringent control to reduce cell volume prior to mitotic entry, PMC is integral and universal for mitosis of mammalian cells (Habela and Sontheimer 2007; Boucrot and Kirchhausen 2008). Given that potassium channels regulate cell volume upon extracellular osmotic challenges, whether potassium channels participate in volume regulation for PMC during mitosis is an interesting open question. We observed a dynamic movement of EAG2 toward the cell membrane upon mitotic entry and a strong increase of the outward potassium current density during MB cell mitosis. This EAG2 enrichment on the cell surface appears to promote $\mathrm{MB}$ cell cycle progression, as evidenced by our knockdown studies. We propose that EAG2 functions on the cell membrane to regulate MB cell volume dynamics via its action on intracellular osmolality. The characteristic noninactivating outward potassium current conducted by EAG2 channel makes it an excellent candidate for reducing intracellular potassium salt concentration and osmolality during channel opening. Consequently, the decrease of intracellular osmolality would drive obligate water efflux, and the loss of cytoplasmic water would eventually lead to a regulated cell volume reduction at late G2 and M phase when EAG2 protein was detected on the cell surface. As the cell volume oscillates during different phases of the cell cycle, it is conceivable that the intracellular osmolality also oscillates according to the cell's necessity to increase or decrease its volume. While EAG2 knockdown resulted in abnormally enlarged cells at late G2 phase, we found that merely increasing the osmolality of the culture medium by adding $\mathrm{NaCl}$ or sucrose could not rescue the EAG2 knockdown phenotype and actually significantly impaired the growth of both the control and EAG2 knockdown MB cells (Supplemental Fig. 10). These results further demonstrate the importance of regulated osmolality changes and cell volume oscillations for successful cell cycle progressions. As we also detected robust Eag2 expression in the proliferative CGNPs during cerebellar development, it would be interesting to determine whether the cell volume regulation by Eag2 uncovered in our study reflects a basic regulatory mechanism for the proliferation of normal precursor cells.

The cell volume decreases as the movement of potassium ions and chloride ions accompanies the water efflux for PMC. In different cancers, different potassium channels or chloride channels may be up-regulated to promote cell proliferation. Consistent with this notion, ClC chloride channels have been shown to localize to the cell membrane and regulate G2-M transition in glioma cells
(Zheng et al. 2002; Habela et al. 2008; Cuddapah et al. 2012). It remains possible that EAG2 in intracellular membrane compartments may also contribute to the regulation of cell cycle progression. Notwithstanding the reported up-regulation of potassium channel expression at the transcript or protein levels in many cancers, it is an open question whether these channels remain at the cell surface and conduct ions throughout the cell cycle. Our study provides an example of dynamic regulation of channel density that increases the activity of an ion channel important for mitotic entry and tumor cell growth. Interestingly, overexpression of another voltage-gated potassium channel, KCNA1 (Kv1.1), specifically marks group 4 MBs (Taylor et al. 2012). Future study is warranted to investigate the underlying mechanism for KCNA1 upregulation and functional importance.

\section{Materials and methods}

\section{Microarray analyses}

Total RNAs were extracted with an RNeasy minikit (Qiagen) from grossly dissected MB tumor tissues of two SmoM2 mice (4 wk and $6 \mathrm{wk}$ ) and one 4-mo-old Ptch ${ }^{\mathrm{LacZ} /+}$ mouse, and the whole cerebellum of one 4-mo-old wild-type mouse. DNase was used to remove genomic DNA. The RNAs then were assessed by electrophoresis and an Agilent 2100 Bioanalyzer to ensure quality before subjecting them to gene expression array using an Affymetrix Mouse Gene 1.0 ST. Gene expression levels and comparisons have been analyzed manually and using DAVID Bioinformatics Resources. The heat map shown in Figure 1A was generated by the Matrix2png program. Microarray results were submitted to NCBI with Gene Expression Omnibus (GEO) submission number GSE36525.

\section{Bioinformatics methods}

As previously described (Northcott et al. 2009), 112 MB samples were hybridized to Affymetrix Genechip Human Exon 1.0ST arrays at the Toronto Center for Applied Genomics (TCAG). Previously performed molecular subgrouping (Northcott et al. $2011 b$ ) permitted the identification of subgroup association for 103 samples. For publicly available human (GSE10327) and mouse (GSE24628) data sets, .CEL files were downloaded and analyzed using Affymetrix Gene Expression Console version 1.1. Following quantile normalization, results were summarized using an RMA algorithm in a similar manner as previously described (Northcott et al. 2009).

\section{Immunohistochemistry}

All immunohistochemistry analyses were performed on tissue sections collected from OCT- or paraffin-embedded tissues. The primary antibodies were rabbit anti-EAG2 (1:500; Sigma), rabbit anti-EAG2 (1:500; LSBio), rabbit anti-EAG2 (1:500; Abcam), mouse anti-Ki67 (1:1000; BD Biosciences), mouse anti-Nestin (1:1000; Millipore), rabbit anti-phospho-histone 3 (1:1000; Millipore), rabbit anti-GFAP (1:1000; Sigma), chicken anti-GFP (1:1000; Aves Labs), and rabbit anti-phospho-p38 (Thr 180/Tyr 182) (1:1000; Cell Signaling).

\section{mRNA in situ hybridization}

Nonradioactive mRNA in situ hybridization analysis was performed on cryosections obtained from snap-frozen tissues. Full- 
Huang et al.

length cDNA of mouse Eag2 was obtained from Open Biosystems (clone ID 30094199) and used as a template for synthesizing digoxygenin-labeled riboprobe.

\section{Archived human MB tumor samples and cell counting}

Paraffin-embedded human MB samples were obtained from the University of California at San Francisco Brain Tumor Research Center Tissue Core. EAG2 antibody targeting different epitopes were obtained from three commercial sources (Sigma, catalog no. HPA030487; Abcam, catalog no. 32975; and LSBio, catalog no. LS-B975) and used for immunohistochemistry experiments with comparable results. More than 500 cells from 10 randomly selected microscopic areas were counted in each patient sample for generating the statistics of EAG2 staining shown in Figure 2D and Supplemental Table 2.

\section{Cell volume measurements and quantifications}

For measuring cell volumes, soluble GFP was overexpressed in the Vandy-MB-11 cells by lentivirus infections and in the COS7 and HEK293 cells by transfections. Confocal images with system-optimized parameters were taken throughout the entire thickness of the cells using a Leica TCS SP5 setup. Then, the confocal LIF files were converted into an Imaris file through ImarisFileConverter 6.4.2. All subsequent image processing was conducted with Imaris 5.5 software. A region of interest encompassing a single cell was chosen for three-dimensional reconstruction and calculating the "Voxel" level for volume quantification. A smooth level of 0.2 was given to every cell for consistency. Volume statistic comparisons were performed with Student's $t$-test.

\section{Acknowledgments}

We thank Yuanquan Song, Andrew Y. Kim, Fen Huang, Peter Soba, and members of the Jan laboratory for helpful discussions. We thank Shaohua Xiao for preparing the cerebellar RNAs from different developmental stages, and Hye Young Lee for help with siRNA transfection and Westerns. We are grateful for stimulating discussion with Anders I. Persson, Sabine Mueller, and Daphne A. Haas-Kogan. We also thank Denan Wang for excellent mouse husbandry, and Joanna Phillips and Cynthia Cowdrey for preparing the paraffin-embedded human tumor samples. This work is supported by a GEMS-CTSI post-doctoral award from the Howard Hughes Medical Institute and University of California at San Francisco, and the Damon Runyon Cancer Research Foundation Fellowship to X.H.; the National Institute of Health grant R37MH065334 to L.Y.J.; and the Pediatric Brain Tumor Foundation and R01s CA133091, CA148699, and CA159859 to W.A.W. and M.D.T. D.H.R., Y.N.J., and L.Y.J. are Howard Hughes Medical Institute investigators.

\section{References}

Arcangeli A, Bianchi L, Becchetti A, Faravelli L, Coronnello M, Mini E, Olivotto M, Wanke E. 1995. A novel inwardrectifying $\mathrm{K}^{+}$current with a cell-cycle dependence governs the resting potential of mammalian neuroblastoma cells. I Physiol 489: 455-471.

Ashcroft FM. 2000. Ion channels and disease: Channelopathies. Academic Press, San Diego.

Ayllon V, O'Connor R. 2007. PBK/TOPK promotes tumour cell proliferation through 38 MAPK activity and regulation of the DNA damage response. Oncogene 26: 3451-3461.
Bloch M, Ousingsawat J, Simon R, Schraml P, Gasser TC, Mihatsch MJ, Kunzelmann K, Bubendorf L. 2007. KCNMA1 gene amplification promotes tumor cell proliferation in human prostate cancer. Oncogene 26: 2525-2534.

Boucrot E, Kirchhausen T. 2008. Mammalian cells change volume during mitosis. PLOS ONE 3: e1477. doi: 10.1371/ journal.pone.0001477.

Buonamici S, Williams J, Morrissey M, Wang A, Guo R, Vattay A, Hsiao K, Yuan J, Green J, Ospina B, et al. 2010. Interfering with resistance to smoothened antagonists by inhibition of the PI3K pathway in medulloblastoma. Sci Transl Med 2: 51ra70. doi: 10.1126/scitranslmed.300159.

Cayabyab FS, Schlichter LC. 2002. Regulation of an ERG $\mathrm{K}^{+}$ current by Src tyrosine kinase. I Biol Chem 277: 1367313681.

Cho YJ, Tsherniak A, Tamayo P, Santagata S, Ligon A, Greulich $\mathrm{H}$, Berhoukim R, Amani V, Goumnerova L, Eberhart CG et al. 2011. Integrative genomic analysis of medulloblastoma identifies a molecular subgroup that drives poor clinical outcome. J Clin Oncol 29: 1424-1430.

Ciemerych MA, Kenney AM, Sicinska E, Kalaszczynska I, Bronson RT, Rowitch DH, Gardner H, Sicinski P. 2002. Development of mice expressing a single D-type cyclin. Genes Dev 16: 3277-3289.

Crociani O, Guasti L, Balzi M, Becchetti A, Wanke E, Olivotto M, Wymore RS, Arcangeli A. 2003. Cell cycle-dependent expression of HERG1 and HERG1B isoforms in tumor cells. J Biol Chem 278: 2947-2955.

Cuddapah VA, Habela CW, Watkins S, Moore LS, Barclay TT, Sontheimer H. 2012. Kinase activation of ClC-3 accelerates cytoplasmic condensation during mitotic cell rounding. Am J Physiol Cell Physiol 302: C527-C538. doi: 10.1152/ajpcell. 00248.2011.

Downie BR, Sanchez A, Knotgen H, Contreras-Jurado C, Gymnopoulos M, Weber C, Stuhmer W, Pardo LA. 2008. Eag1 expression interferes with hypoxia homeostasis and induces angiogenesis in tumors. J Biol Chem 283: 36234-36240.

Ellison DW, Dalton J, Kocak M, Nicholson SL, Fraga C, Neale G, Kenney AM, Brat DJ, Perry A, Yong WH, et al. 2011. Medulloblastoma: Clinicopathological correlates of $\mathrm{SHH}$, WNT, and non-SHH/WNT molecular subgroups. Acta Neuropathol 121: 381-396.

Fraser SP, Pardo LA. 2008. Ion channels: Functional expression and therapeutic potential in cancer. Colloquium on ion channels and cancer. EMBO Rep 9: 512-515.

Fraser SP, Grimes JA, Diss JK, Stewart D, Dolly JO, Diamgoz MB. 2003. Predominant expression of Kv1.3 voltage-gated $\mathrm{K}^{+}$ channel subunit in rat prostate cancer cell lines: Electrophysiological, pharmacological and molecular characterisation. Pflugers Arch 446: 559-571.

Ganetzky B, Robertson GA, Wilson GF, Trudeau MC, Titus SA. 1999. The eag family of $\mathrm{K}^{+}$channels in Drosophila and mammals. Ann NY Acad Sci 868: 356-369.

Gibson P, Tong Y, Robinson G, Thompson MC, Currle DS, Eden C, Kranenburg TA, Hogg T, Poppleton H, Martin J, et al. 2010. Subtypes of medulloblastoma have distinct developmental origins. Nature 468: 1095-1099.

Goncalves JT, Stuhmer W. 2010. Calmodulin interaction with hEAG1 visualized by FRET microscopy. PLOS ONE 5: e10873. doi: 10.1371/journal.pone.0010873.

Goodrich LV, Milenkovic L, Higgins KM, Scott MP. 1997. Altered neural cell fates and medulloblastoma in mouse patched mutants. Science 277: 1109-1113.

Guldal CG, Ahmad A, Korshunov A, Squatrito M, Awan A, Mainwaring LA, Bhatia B, Parathath SR, Nahle Z, Pfister S, et al. 2012. An essential role for p38 MAPK in cerebellar 
granule neuron precursor proliferation. Acta Neuropathol 123: $573-586$.

Habela CW, Sontheimer H. 2007. Cytoplasmic volume condensation is an integral part of mitosis. Cell Cycle 6: 1613-1620.

Habela CW, Olsen ML, Sontheimer H. 2008. ClC3 is a critical regulator of the cell cycle in normal and malignant glial cells. J Neurosci 28: 9205-9217.

Han J, Sun P. 2007. The pathways to tumor suppression via route p38. Trends Biochem Sci 32: 364-371.

Hatton BA, Villavicencio EH, Tsuchiya KD, Pritchard JI, Ditzler S, Pullar B, Hansen S, Knoblaugh SE, Lee D, Eberhart CG, et al. 2008. The Smo/Smo model: hedgehog-induced medulloblastoma with $90 \%$ incidence and leptomeningeal spread. Cancer Res 68: 1768-1776.

Hegle AP, Marble DD, Wilson GF. 2006. A voltage-driven switch for ion-independent signaling by ether-a-go-go $\mathrm{K}^{+}$channels. Proc Natl Acad Sci 103: 2886-2891.

Hemmerlein B, Weseloh RM, Mello de Queiroz F, Knotgen H, Sanchez A, Rubio ME, Martin S, Schliephacke T, Jenke M, Heinz Joachim R, et al. 2006. Overexpression of Eag1 potassium channels in clinical tumours. Mol Cancer 5: 41. doi: 10.1186/1476-4598-5-41.

Hille B. 2001. Ion channels of excitable membranes. Sinauer, Sunderland, Mass.

Jehle J, Schweizer PA, Katus HA, Thomas D. 2011. Novel roles for hERG K ${ }^{+}$channels in cell proliferation and apoptosis. Cell Death Dis 2: e193. doi: 10.1038/cddis.2011.77.

Kaplan WD, Trout WE 3rd. 1969. The behavior of four neurological mutants of Drosophila. Genetics 61: 399-409.

Kawauchi D, Robinson G, Uziel T, Gibson P, Rehg J, Gao C, Finkelstein D, Qu C, Pounds S, Ellison DW, et al. 2012. A mouse model of the most aggressive subgroup of human medulloblastoma. Cancer Cell 21: 168-180.

Kenney AM, Rowitch DH. 2000. Sonic hedgehog promotes G(1) cyclin expression and sustained cell cycle progression in mammalian neuronal precursors. Mol Cell Biol 20: 90559067.

Kenney AM, Cole MD, Rowitch DH. 2003. Nmyc upregulation by sonic hedgehog signaling promotes proliferation in developing cerebellar granule neuron precursors. Development 130: 15-28.

Kim CJ, Cho YG, Jeong SW, Kim YS, Kim SY, Nam SW, Lee SH, Yoo NJ, Lee JY, Park WS. 2004. Altered expression of KCNK9 in colorectal cancers. APMIS 112: 588-594.

Kimura H, Ng JM, Curran T. 2008. Transient inhibition of the Hedgehog pathway in young mice causes permanent defects in bone structure. Cancer Cell 13: 249-260.

Kool M, Koster J, Bunt J, Hasselt NE, Lakeman A, van Sluis P, Troost D, Meeteren NS, Caron HN, Cloos J, et al. 2008. Integrated genomics identifies five medulloblastoma subtypes with distinct genetic profiles, pathway signatures and clinicopathological features. PLOS ONE 3: e3088. doi: 10.1371/ journal.pone.0003088.

Kumar S, Jiang MS, Adams JL, Lee JC. 1999. Pyridinylimidazole compound SB 203580 inhibits the activity but not the activation of p38 mitogen-activated protein kinase. Biochem Biophys Res Commun 263: 825-831.

Liu X, Chang Y, Reinhart PH, Sontheimer H. 2002. Cloning and characterization of glioma $\mathrm{BK}$, a novel $\mathrm{BK}$ channel isoform highly expressed in human glioma cells. J Neurosci 22: 18401849.

Liu H, Zhang H, Jiang X, Ma Y, Xu Y, Feng S, Liu F. 2011. Knockdown of secreted protein acidic and rich in cysteine (SPARC) expression diminishes radiosensitivity of glioma cells. Cancer Biother Radiopharm 26: 705-715.

Lu C, Shi Y, Wang Z, Song Z, Zhu M, Cai Q, Chen T. 2008. Serum starvation induces $\mathrm{H} 2 \mathrm{AX}$ phosphorylation to regulate apoptosis via p38 MAPK pathway. FEBS Lett 582: 27032708.

Ludwig J, Weseloh R, Karschin C, Liu Q, Netzer R, Engeland B, Stansfeld C, Pongs O. 2000. Cloning and functional expression of rat eag2, a new member of the ether-a-go-go family of potassium channels and comparison of its distribution with that of eag1. Mol Cell Neurosci 16: 59-70.

Mao J, Ligon KL, Rakhlin EY, Thayer SP, Bronson RT, Rowitch D, McMahon AP. 2006. A novel somatic mouse model to survey tumorigenic potential applied to the Hedgehog pathway. Cancer Res 66: 10171-10178.

Marshall WS, Ossum CG, Hoffmann EK. 2005. Hypotonic shock mediation by $\mathrm{p} 38$ MAPK, JNK, PKC, FAK, OSR1 and SPAK in osmosensing chloride secreting cells of killifish opercular epithelium. J Exp Biol 208: 1063-1077.

McManamy CS, Pears J, Weston CL, Hanzely Z, Ironside JW, Taylor RE, Grundy RG, Clifford SC, Ellison DW. 2007. Nodule formation and desmoplasia in medulloblastomasdefining the nodular/desmoplastic variant and its biological behavior. Brain Pathol 17: 151-164.

Mello de Queiroz F, Suarez-Kurtz G, Stuhmer W, Pardo LA. 2006. Ether a go-go potassium channel expression in soft tissue sarcoma patients. Mol Cancer 5: 42.

Meyer R, Heinemann SH. 1998. Characterization of an eag-like potassium channel in human neuroblastoma cells. I Physiol 508: 49-56

Mu D, Chen L, Zhang X, See LH, Koch CM, Yen C, Tong JJ, Spiegel L, Nguyen KC, Servoss A, et al. 2003. Genomic amplification and oncogenic properties of the KCNK9 potassium channel gene. Cancer Cell 3: 297-302.

Northcott PA, Nakahara Y, Wu X, Feuk L, Ellison DW, Croul S, Mack S, Kongkham PN, Peacock J, Dubuc A, et al. 2009. Multiple recurrent genetic events converge on control of histone lysine methylation in medulloblastoma. Nat Genet 41: 465-472.

Northcott PA, Hielscher T, Dubuc A, Mack S, Shih D, Remke M, Al-Halabi H, Albrecht S, Jabado N, Eberhart CG, et al. 2011a. Pediatric and adult sonic hedgehog medulloblastomas are clinically and molecularly distinct. Acta Neuropathol 122: 231-240.

Northcott PA, Korshunov A, Witt H, Hielscher T, Eberhart CG, Mack S, Bouffet E, Clifford SC, Hawkins CE, French P et al. 2011b. Medulloblastoma comprises four distinct molecular variants. J Clin Oncol 29: 1408-1414.

Pardo LA. 2004. Voltage-gated potassium channels in cell proliferation. Physiology (Bethesda) 19: 285-292.

Pardo LA, Bruggemann A, Camacho J, Stuhmer W. 1998. Cell cycle-related changes in the conducting properties of r-eag $\mathrm{K}^{+}$ channels. J Cell Biol 143: 767-775.

Pardo LA, del Camino D, Sanchez A, Alves F, Bruggemann A, Beckh S, Stuhmer W. 1999. Oncogenic potential of EAG $\mathrm{K}^{+}$ channels. EMBO J 18: 5540-5547.

Pardo LA, Contreras-Jurado C, Zientkowska M, Alves F, Stuhmer W. 2005. Role of voltage-gated potassium channels in cancer. J Membr Biol 205: 115-124.

Parsons DW, Li M, Zhang X, Jones S, Leary RJ, Lin JC, Boca SM, Carter H, Samayoa J, Bettegowda C, et al. 2011. The genetic landscape of the childhood cancer medulloblastoma. Science 331: 435-439.

Pei Y, Moore CE, Wang J, Tewari AK, Eroshkin A, Cho YJ, Witt H, Korshunov A, Read TA, Sun JL, et al. 2012. An animal model of MYC-driven medulloblastoma. Cancer Cell 21: 155-167.

Pillozzi S, Brizzi MF, Balzi M, Crociani O, Cherubini A, Guasti L, Bartolozzi B, Becchetti A, Wanke E, Bernabei PA, et al. 2002. HERG potassium channels are constitutively expressed 
in primary human acute myeloid leukemias and regulate cell proliferation of normal and leukemic hemopoietic progenitors. Leukemia 16: 1791-1798.

Prevarskaya N, Skryma R, Shuba Y. 2010. Ion channels and the hallmarks of cancer. Trends Mol Med 16: 107-121.

Romer J, Curran T. 2005. Targeting medulloblastoma: Smallmolecule inhibitors of the Sonic Hedgehog pathway as potential cancer therapeutics. Cancer Res 65: 4975-4978.

Rudin CM, Hann CL, Laterra J, Yauch RL, Callahan CA, Fu L, Holcomb T, Stinson J, Gould SE, Coleman B, et al. 2009. Treatment of medulloblastoma with hedgehog pathway inhibitor GDC-0449. N Engl J Med 361: 1173-1178.

Salsano E, Pollo B, Eoli M, Giordana MT, Finocchiaro G. 2004. Expression of MATH1, a marker of cerebellar granule cell progenitors, identifies different medulloblastoma sub-types. Neurosci Lett 370: 180-185.

Salsano E, Croci L, Maderna E, Lupo L, Pollo B, Giordana MT, Consalez GG, Finocchiaro G. 2007. Expression of the neurogenic basic helix-loop-helix transcription factor NEUROG1 identifies a subgroup of medulloblastomas not expressing ATOH1. Neuro-oncol 9: 298-307.

Schonherr R, Lober K, Heinemann SH. 2000. Inhibition of human ether a go-go potassium channels by $\mathrm{Ca}^{2+} /$ calmodulin. EMBO I 19: 3263-3271.

Schuller U, Heine VM, Mao J, Kho AT, Dillon AK, Han YG, Huillard E, Sun T, Ligon AH, Qian Y, et al. 2008. Acquisition of granule neuron precursor identity is a critical determinant of progenitor cell competence to form Shh-induced medulloblastoma. Cancer Cell 14: 123-134.

Stringer BK, Cooper AG, Shepard SB. 2001. Overexpression of the G-protein inwardly rectifying potassium channel 1 (GIRK1) in primary breast carcinomas correlates with axillary lymph node metastasis. Cancer Res 61: 582-588.

Swartling FJ, Grimmer MR, Hackett CS, Northcott PA, Fan QW, Goldenberg DD, Lau J, Masic S, Nguyen K, Yakovenko S, et al. 2010. Pleiotropic role for MYCN in medulloblastoma. Genes Dev 24: 1059-1072.

Swartling FJ, Savov V, Persson AI, Chen J, Hackett CS, Northcott PA, Grimmer MR, Lau J, Chesler L, Perry A, et al. 2012. Distinct neural stem cell populations give rise to disparate brain tumors in response to N-MYC. Cancer Cell 21: 601-613.

Taylor MD, Northcott PA, Korshunov A, Remke M, Cho YJ, Clifford SC, Eberhart CG, Parsons DW, Rutkowski S, Gajjar A, et al. 2012. Molecular subgroups of medulloblastoma: The current consensus. Acta Neuropathol 123: 465-472.

Thompson MC, Fuller C, Hogg TL, Dalton J, Finkelstein D, Lau CC, Chintagumpala M, Adesina A, Ashley DM, Kellie SJ et al. 2006. Genomics identifies medulloblastoma subgroups that are enriched for specific genetic alterations. J Clin Oncol 24: 1924-1931.

Wagner EF, Nebreda AR. 2009. Signal integration by JNK and p38 MAPK pathways in cancer development. Nat Rev Cancer 9: 537-549.

Warmke J, Drysdale R, Ganetzky B. 1991. A distinct potassium channel polypeptide encoded by the Drosophila eag locus. Science 252: 1560-1562.

Weber C, Mello de Queiroz F, Downie BR, Suckow A, Stuhmer W, Pardo LA. 2006. Silencing the activity and proliferative properties of the human EagI potassium channel by RNA interference. J Biol Chem 281: 13030-13037.

Wechsler-Reya RJ, Scott MP. 1999. Control of neuronal precursor proliferation in the cerebellum by Sonic Hedgehog. Neuron 22: 103-114.

Wlodarski P, Grajkowska W, Lojek M, Rainko K, Jozwiak J. 2006. Activation of Akt and Erk pathways in medulloblastoma. Folia Neuropathol 44: 214-220.
Wonderlin WF, Strobl JS. 1996. Potassium channels, proliferation and G1 progression. J Membr Biol 154: 91-107.

Yang ZJ, Ellis T, Markant SL, Read TA, Kessler JD, Bourboulas M, Schuller U, Machold R, Fishell G, Rowitch DH, et al. 2008. Medulloblastoma can be initiated by deletion of Patched in lineage-restricted progenitors or stem cells. Cancer Cell 14: 135-145.

Yang F, Jove V, Xin H, Hedvat M, Van Meter TE, Yu H. 2010. Sunitinib induces apoptosis and growth arrest of medulloblastoma tumor cells by inhibiting STAT3 and AKT signaling pathways. Mol Cancer Res 8: 35-45.

Yauch RL, Dijkgraaf GJ, Alicke B, Januario T, Ahn CP, Holcomb T, Pujara K, Stinson J, Callahan CA, Tang T, et al. 2009. Smoothened mutation confers resistance to a Hedgehog pathway inhibitor in medulloblastoma. Science 326: 572574.

Zheng YJ, Furukawa T, Ogura T, Tajimi K, Inagaki N. 2002. M phase-specific expression and phosphorylation-dependent ubiquitination of the ClC-2 channel. I Biol Chem 277: 32268-32273.

Zhou BB, Elledge SJ. 2000. The DNA damage response: Putting checkpoints in perspective. Nature 408: 433-439.

Ziechner U, Schonherr R, Born AK, Gavrilova-Ruch O, Glaser RW, Malesevic M, Kullertz G, Heinemann SH. 2006. Inhibition of human ether a go-go potassium channels by $\mathrm{Ca}^{2+} /$ calmodulin binding to the cytosolic $\mathrm{N}$ - and $\mathrm{C}$-termini. FEBS $J$ 273: 1074-1086. 


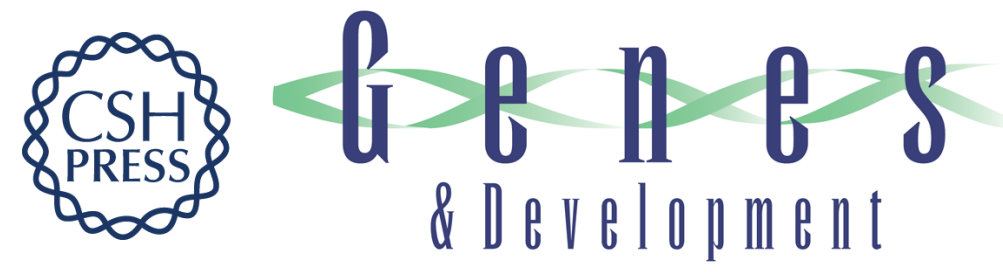

\section{Voltage-gated potassium channel EAG2 controls mitotic entry and tumor growth in medulloblastoma via regulating cell volume dynamics}

Xi Huang, Adrian M. Dubuc, Rintaro Hashizume, et al.

Genes Dev. 2012, 26: originally published online August 1, 2012

Access the most recent version at doi:10.1101/gad.193789.112

\section{Supplemental http://genesdev.cshlp.org/content/suppl/2012/07/25/gad.193789.112.DC1 Material}

References This article cites 78 articles, 29 of which can be accessed free at: http://genesdev.cshlp.org/content/26/16/1780.full.html\#ref-list-1

\section{License}

Email Alerting

Receive free email alerts when new articles cite this article - sign up in the box at the top Service

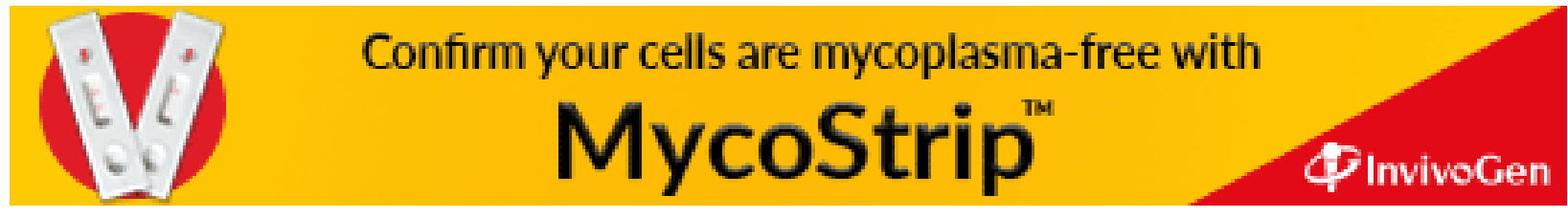

